\title{
90 years of thermal analysis as a control tool in the melting of cast iron
}

\author{
*Doru Michael Stefanescu ${ }^{1}$, Ramon Suarez ${ }^{2,3}$, and Sung Bin Kim ${ }^{4}$ \\ 1. The Ohio State University, Columbus, $\mathrm{OH}$ and The University of Alabama, Tuscaloosa, AL, USA \\ 2. IK4-Azterlan, Durango (Bizkaia), Spain \\ 3. Veigalan Estudio 2010, Durango (Bizkaia), Spain \\ 4. AnyCasting Software Co., Ltd, Seoul, S. Korea
}

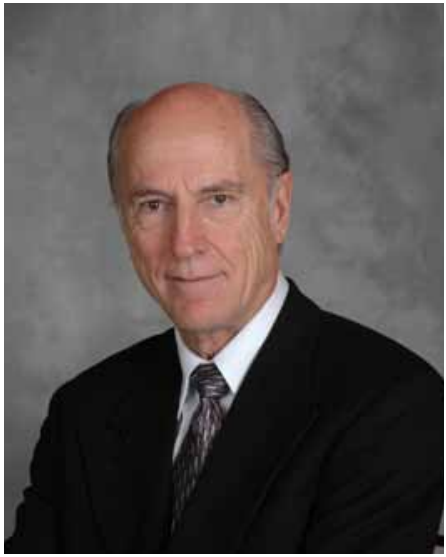

*Doru Michael Stefanescu

Professor Stefanescu, a graduate of the University Politehnica Bucharest, Romania, served as a Visiting Professor at the Univ. of Wisconsin - Madison (1980), and then joined the Univ. of Alabama (UA) where he taught and did research until 2005. In August of 2005, he joined the Material Science and Engineering Department at the Ohio State Univ. (OSU) until August 2010. Prof. Stefanescu's research interests include experimental and numerical aspects of solidification processing, influence of low-gravity on solidification, processing of metal-matrix composites manufacturing technologies and physical metallurgy of cast iron, steel and nonferrous alloys, etc. The research area in which his accomplishments have probably been most widely recognized nationally and internationally is computational modeling of microstructure evolution during solidification of castings. Another area of significant contribution is that of particles' behavior at the liquid/solid interface. Professor Stefanescu is recognized as a world expert in cast iron. He is the author of over 447 scientific and technical publications. Prof. Stefanescu is the editor of ASM Handbook Vol. 1A Cast Iron Science and Technology, ASM International (2017). He is a Dr. Honoris Causa of three universities and an Honorary Member of the Romanian Academy of Technical Sciences. Prof. Stefanescu awards include the Gold Medal and Honorary Life membership by the American Foundry Society, four NASA Certificates of Recognition for the Creative Development of Technical Innovations, the John Campbell Medal, Institute of Cast Metals Engineers, United Kingdom, and others.

E-mail: stefanescu.1@osu.edu

Received: 2020-03-13; Accepted: 2020-03-25
Abstract: Since its first literature mention in conjunction with cast iron in 1931 by Esser and Lautenbusch, thermal analysis (TA) has journeyed a long way. Today it is an accepted and widely used tool for process control for all types of cast irons. This paper reviews the latest progress in the development of equipment and analysis methods that make TA successful in applications such as the estimation of chemical composition, graphitization potential, and the shape and number of graphite aggregates. The potential and limitations of the prediction of shrinkage defects propensity are analyzed in some details. Examples of attempts at prediction of mechanical properties and shrinkage propensity are also discussed. Several graphs showing the data scattering are presented to convey the reader a better sense of the accuracy of various predictions.

Key words: thermal analysis; cast iron; graphite; shrinkage prediction; chemical composition

CLC numbers: TG143; Document code: A;

Article ID: 1672-6421(2020)02-069-016

$\mathrm{W}$ hile the first temperature-time record of heating curves appears to have been reported as early as $1877^{[1]}$, it was not until 1931 that the method was applied to cast iron analysis by Esser and Lautenbusch, cited by Piwowarski ${ }^{[2]}$. They reported that higher superheating of gray iron depresses the temperature of eutectic arrest. Other qualitative observations include those by Piwowarski ${ }^{[3]}$ who noticed that higher silicon decreases the undercooling, by Loper et al. ${ }^{[4]}$ who reported the effect of the $\mathrm{Mn} / \mathrm{S}$ ratio on the eutectic undercooling, by Naro and Wallace ${ }^{[5]}$ who revealed the effect of Ce and S additions, and by De Sy and Vidts ${ }^{[6]}$ who pointed out that cooling curves contain information on graphite morphology (shape).

In time, thermal analysis (TA), the technique of recording and interpretation of cooling curves, became an important tool in online monitoring of melt quality, a tool that proved to be of paramount importance for quality assurance of castings. While initial applications of termed TA revolved around the rapid evaluation of carbon equivalent in cast iron, or of the silicon content in Al-Si alloys, further developments extended the pertinency of the method to the evaluation of the effect of chemical composition and cooling rate variations on the outcome of solidification microstructure. Then, as computational approaches were facilitated by ever faster computers, the use and interpretation of the time-derivatives of the cooling curve made possible prediction of the 
nucleation potential (degree of inoculation) and basic elements of the solidification microstructure such as dendrite arm spacing, grain size, graphite morphology, and room-temperature microstructure. More recently, attempts at predicting the shrinkage propensity firmly established TA as a basic tool in process control of all grades of cast irons. The main reason for this development is the observation that consistent melt chemistry is not sufficient to ensure that the casting meets the target properties. Consequently, TA is now used as a dynamic process control tool as it allows anticipating the solidification behavior $^{[7]}$.

This review is an update of an earlier one ${ }^{[8]}$. Endeavoring to avoid duplication, we will refer occasionally to this previous work, while emphasizing the latest developments.

\section{Principles of thermal analysis}

In its simplest form, TA, also known to the foundrymen as Cooling Curve Analysis (CCA), consists in recording the temperature measured by one or several thermocouples inserted in the mold. The mold may be a standard cup or the casting of interest. The iron may be poured in a sand cup (Fig. 1a) or in a metal cup that will be immersed in the metal to collect a sample, and then extracted from the melt and allowed to cool and solidify (Fig. 1b). As the design of the test cup has a significant effect on the results and their interpretation, the issue of cup design will be further discussed later in more detail. The shape of the cooling curve is determined by the balance between the latent heat liberated during solidification and the heat lost to surroundings (the test cup and the atmosphere).

More recently, Anjos et al. ${ }^{[10]}$ developed a dualcup TA system where in one chamber the hot junction of the thermocouple was placed at the center, and in the other chamber the hot junction was close to the wall of the cup, as presented in Fig. 2. One of its main advantages is in producing cups with constant weight,
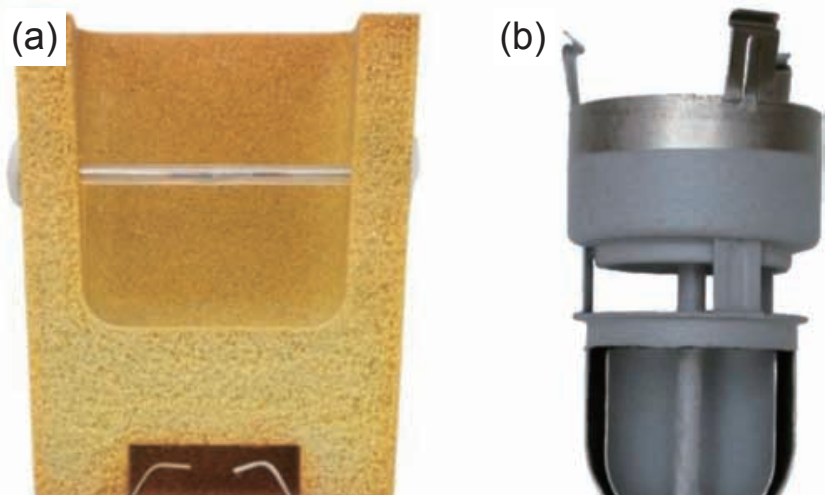

Fig. 1: Examples of test cups for cooling curve analysis: (a) sand cup with disposable thermocouple (Electro-Nite); (b) steel cup (SinterCast) with two thermocouples in the protective tube ${ }^{[9]}$
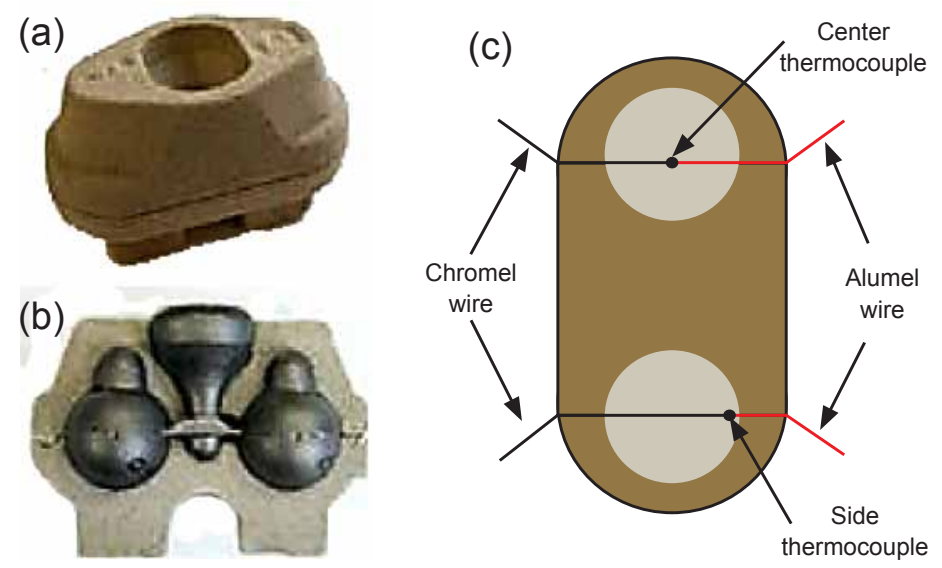

Fig. 2: GmbH dual-cup thermal analysis mold: (a) external view of the cup; (b) image from the internal sample body shape; (c) top view of the bottom half of the cup showing the disposition of the two thermocouples ${ }^{[10]}$

which improves the reliability of the interpretation of the TA.

The cooling curves in Fig. 3(a), adapted from Stefanescu ${ }^{[1]}$, demonstrate the differences occurring during the solidification of four irons with similar carbon equivalent (CE). It is noticed that the white iron has the highest undercooling and that the lamellar graphite (LG) iron has the lowest. Also, compacted graphite (CG) iron has a higher maximum undercooling than spheroidal graphite (SG) iron. (a)

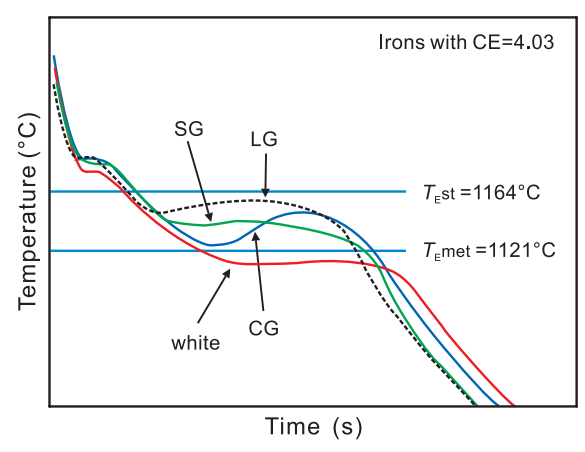

(b)

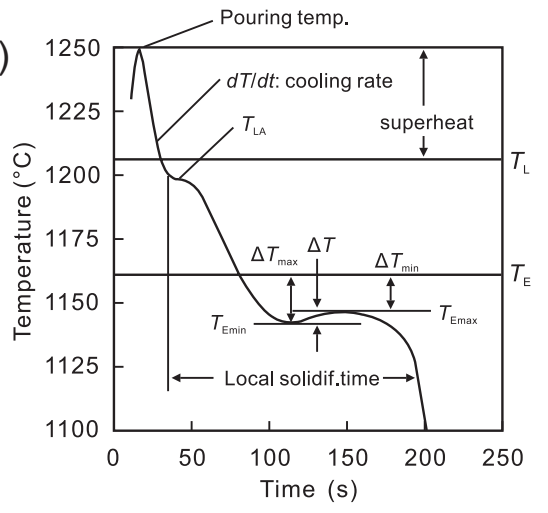

Fig. 3: Cooling curves for irons poured in Electro-Nite type sand cups: (a) cooling curves for four irons with similar carbon equivalent; (b) cooling curve and standard terminologies ${ }^{[8]}$ 
The standard terminologies used in CCA are introduced in Fig. 3(b) and summarized in Table 1. The solidification time can be found from the time difference between the start and end of solidification, and a cooling rate can be calculated for a given temperature from the slope of the cooling curve at that temperature. The second column in Table 1, quoted from Persson et al. ${ }^{[12]}$, is that used by the commercial software ATAS.

Table 1: Most common terminologies used to define important parameters of the cooling curve

\begin{tabular}{|c|c|c|}
\hline \multirow{2}{*}{ Parameter } & \multicolumn{2}{|c|}{ Symbol } \\
\hline & Ref. $[8,13]$ & Ref. [12] \\
\hline Equilibrium liquidus temperature & $T_{\mathrm{L}}$ & \\
\hline Temperature of liquidus arrest & $T_{\mathrm{LA}}$ & $T L$ \\
\hline Equilibrium eutectic temperature & $T_{\mathrm{E}}$ & $T E$ \\
\hline Start of eutectic solidification & & TE_start \\
\hline Temperature of eutectic undercooling & $T_{\text {Emin }}$ & TE_low \\
\hline Temperature of eutectic recalescence & $T_{\text {Emax }}$ & TE_high \\
\hline Recalescence & $\Delta T$ & \\
\hline Maximum undercooling & $\Delta T_{\max }$ & \\
\hline Minimum undercooling & $\Delta T_{\min }$ & \\
\hline Temperature of end of solidification & $T_{\mathrm{S}}$ & TS \\
\hline Min. cooling rate during eutectic reaction (max. of 1 st derivative) & $(d T / d t)_{\min }$ & \\
\hline Max. cooling rate after eutectic reaction (min. of 1 st derivative) & $(d T / d t)_{\max }$ & \\
\hline Time interval at the end of eutectic reaction (on 2 nd derivative) & $\Delta t_{\text {end }}$ & \\
\hline
\end{tabular}

Some basic concepts used in the following text are explained with the help of Fig. 4. It should be noted that only the parameters $T E \_l o w$ and $T E \_$high can be determined directly from the cooling curve. Before the beginning of solidification $(T>T L)$ the molten metal in the cup contracts, producing liquid shrinkage. Solidification then starts at $T L$ (or $T_{\mathrm{LA}}$ ) with formation of dendrites that grow inward from the walls of the cup until the start of the eutectic solidification (Zone 1 on the figure). In a hypoeutectic iron, immediately after the

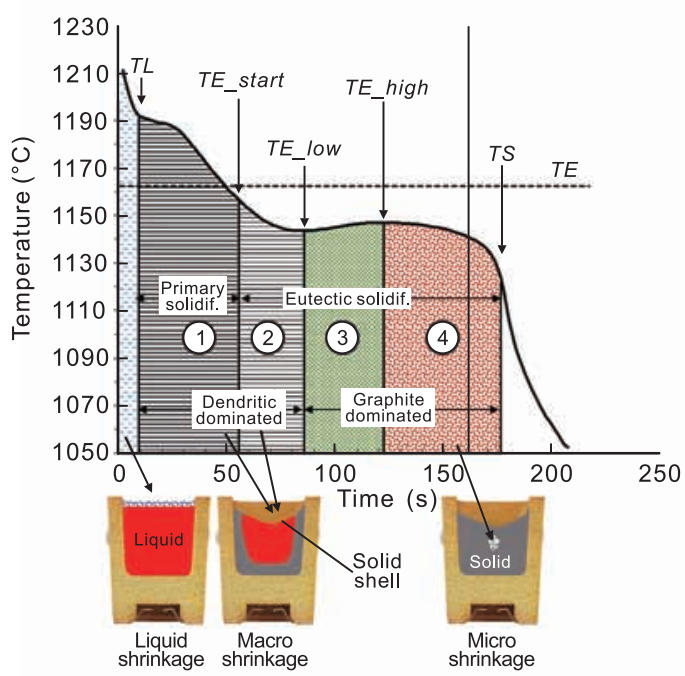

Fig. 4: Cooling curve of a hypoeutectic iron with characteristic temperatures showing solidification ranges for primary and eutectic phases and correlation with shrinkage defects formation start of the eutectic solidification, growth is still dominated by the dendrites, as the amount of eutectics produced increases slowly over time (Zone 2). After the maximum undercooling is reached (TE_low), the increasing amount of eutectic generated shifts the emphasis of solidification from dendritic dominated (Zones 1 and 2) to graphite dominated (Zones 3 and 4). In Zone 3, graphite expansion compensates for liquid and dendrite shrinking. However, in Zone 4, when the amount of eutectic generated and thus that of the graphite decreases, there is a risk of microshrinkage (microporosity) formation, as graphite expansion may become insufficient to compensate for the shrinkage.

More accurate determination of the values of the beginning and end of solidification events from the cooling curve became possible only after the introduction of computer generated cooling rates from cooling curve data (first derivative of the temperature-time curve) by Rabus and Polten ${ }^{[14]}$ in 1972. A second derivative of the cooling curve was then calculated and studied ${ }^{[15]}$. A summary of the parameters of interest is illustrated in Fig. 5 (see Table 1 for terminology). Then, higher order derivatives, as high as the 5 th derivative by Sparkman ${ }^{[16]}$, were interpreted with more or less success. While the physical meaning of the first and second derivatives is clear, that of the higher order derivatives is less understood.

To precisely determine the values of the beginning and end of solidification events from the cooling curve, differential thermal analysis (DTA) must be used. Classic DTA is performed with a reference body ${ }^{[8]}$. However, for in-line process control in metal casting, DTA without a reference body was developed based on 


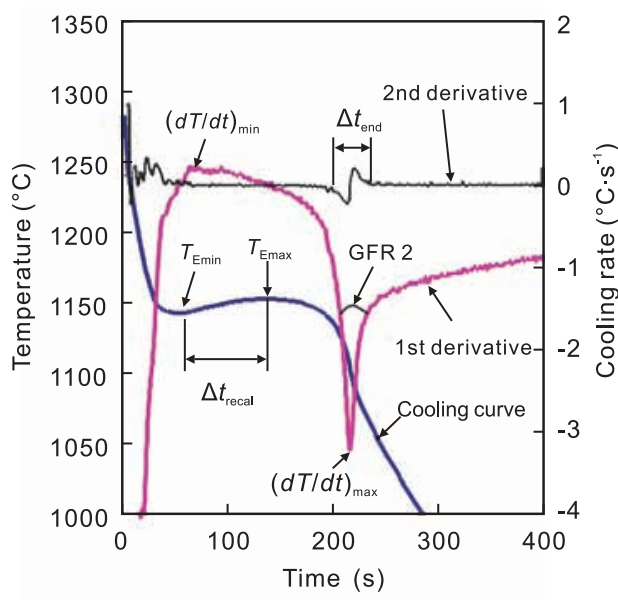

Fig. 5: Cooling curve and its first and second derivatives ${ }^{[13]}$

the calculation of the derivatives of the cooling curve (computeraided cooling curve analysis), as integral calculus applied to temperature-time data allows evaluation of areas that are directly related to the energy evolution. There are, in principle, two major approaches: (1) Newtonian analysis and (2) Fourier analysis. Newtonian analysis requires only one thermocouple and is the most widely used. Fourier analysis is a more accurate treatment of the heat transfer problem, but it requires two thermocouples and the mathematics is more cumbersome ${ }^{[8]}$.

The Newtonian method consists in generating the $1 \mathrm{st}$ derivative of the cooling curve with respect to time (the cooling rate), generating a zero-curve (the cooling without transformation), and then subtracting the area under the zerocurve from the area under the cooling rate. Then, the evolution in time of the amount of phases is calculated as the ratio between the area corresponding to a phase formation (e.g. area corresponding to the solidification of primary austenite) to the total area, as illustrated in Fig. 6. Mathematically, this is represented by the equation:

$$
f_{\mathrm{s}}^{t}=\frac{Q_{\mathrm{f}}^{t}}{Q_{\mathrm{f}}}=\frac{v \rho c_{\mathrm{p}} \int_{t_{0}}^{t}\left[(d T / d t)_{\mathrm{cc}}-(d T / d t)_{\mathrm{zc}}\right]}{Q_{\mathrm{f}}}
$$

where $f_{\mathrm{s}}^{t}$ is the fraction solid generated till time $t, Q_{\mathrm{f}}$ is the total latent heat of fusion, $Q_{\mathrm{f}}^{t}$ is the heat of fusion generated till time $t$,

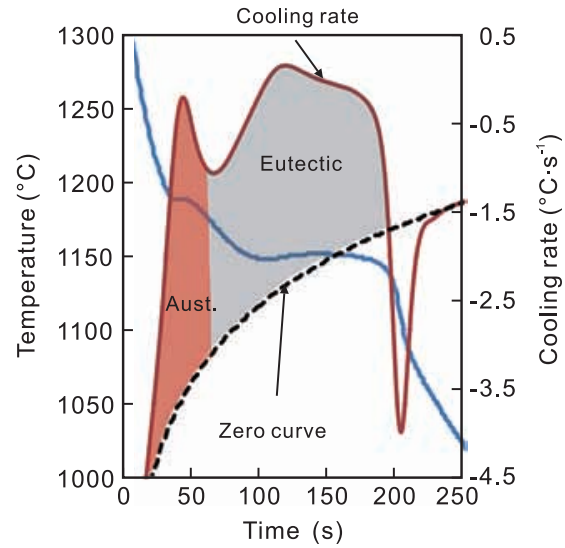

Fig. 6: Calculation of amount of phases from the areas under the cooling rate and the zero curves; the shaded areas are proportional to latent heat released $v$ is the sample volume, $\rho$ and $c_{\mathrm{p}}$ are the density and the specific heat of the alloy, respectively, $t_{\mathrm{o}}$ is the initial time, $d T / d t$ is the cooling rate and the subscripts cc and zc designate the cooling curve and the zero curve, respectively.

Unfortunately, there is no unique accepted method to obtain the zero-curve because of the limitations of the Newtonian analysis. The main assumption of the method is that the thermal gradient across the sample is zero. Consequently, it is limited to Biot number less than 0.1, where the Biot number is $B i=h / k \cdot v / A$, where $h$ is the heat transfer coefficient, $k$ is the thermal conductivity, $v$ is the volume, and $A$ is the cooling area ${ }^{[17]}$. It is thus reasonable for aluminum alloys, but less so for cast iron. The second difficulty is that heat transfer between the casting and the mold is assumed to be by convection and therefore, calculations rely on the heat transfer coefficient which continuously changes during solidification. As a consequence, there is no unique method to calculate the zero-curve. Different approaches have been proposed by a number of investigators ${ }^{[18-21]}$.

To establish the beginning and end of phase transformations, most current commercial software programs use the first derivative of the cooling curve. However, as discussed in detail by Alonso et al. ${ }^{[22]}$, a correct evaluation of these temperatures must be done using the second derivative. This is supported by the example in Fig. 7, where it is seen that the maximum of the 2nd derivative corresponds to the beginning of the austenite solidification, while the maximum of the 1st derivative indicates the liquidus arrest temperature $\left(T_{\mathrm{LA}}\right)$. Also, the minimum on the 2nd derivative corresponds to the end of the eutectic solidification (Fig. 8). When using the 1st derivative, the end of solidification is calculated to occur at unreasonably low temperatures.

A more accurate mathematical approach to the problem is the Fourier analysis which assumes heat transfer by conduction. It requires two thermocouples in the test casting. The Fourier equation with a heat source term is ${ }^{[23,24]}$ :

$$
\frac{\partial T}{\partial t}=\propto \nabla^{2} T+\frac{1}{c_{\mathrm{p}}} \frac{\partial Q_{\mathrm{f}}}{\partial t} \quad \text { or } \quad \frac{\partial Q_{\mathrm{f}}}{\partial t}=c_{\mathrm{p}}\left(\frac{\partial T}{\partial t}-Z_{\mathrm{F}}\right)
$$

with the zero-curve given by $Z_{\mathrm{F}}=\propto \nabla^{2} T$. To calculate this curve, the temperature field must be known, which for a

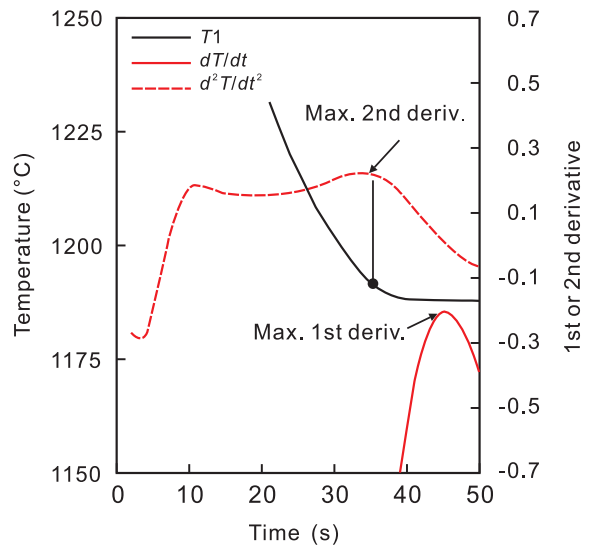

Fig. 7: Use of the 2nd derivative to establish the beginning of primary (austenite) solidification for a gray iron ${ }^{[22]}$ 


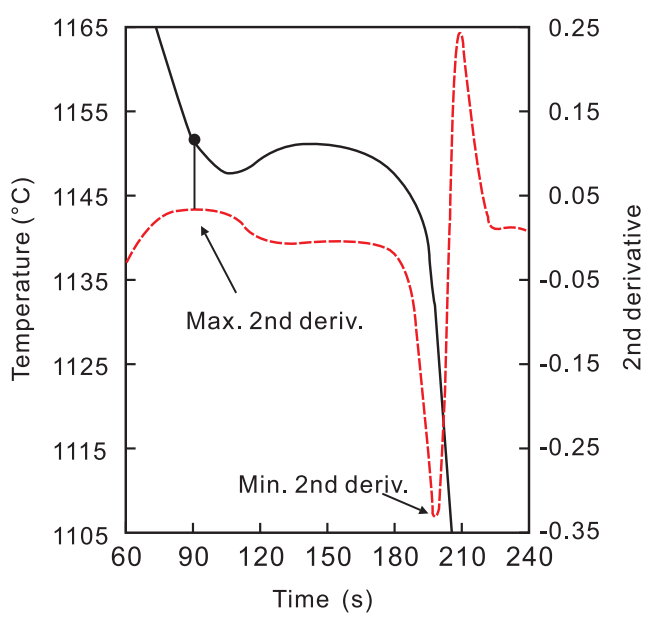

Fig. 8: Use of the 2nd derivative to establish the beginning and the end of eutectic solidification ${ }^{[22]}$

cylindrical mold can be calculated as $\nabla^{2} T=4\left(T_{2}-T_{1}\right) /\left(r_{2}{ }^{2}-r_{1}{ }^{2}\right)$, where $T_{2}$ and $T_{1}$ are the temperatures at radii $r_{2}$ and $r_{1}$, respectively. The latent heat and fraction solid evolution are calculated as described for the Newtonian analysis. Typical results are shown in Fig. 9 for an aluminum alloy. The time close to the end of solidification, when the cooling curve and the Fourier zero curve start coinciding, is considered the end of solidification. Note that this occurs earlier than the minimum at the end of the cooling curve. For a more detailed analysis of the Fourier method through inverse heat conduction analysis, the reader is referred to the paper by Diószegi and Hattel ${ }^{[25]}$.

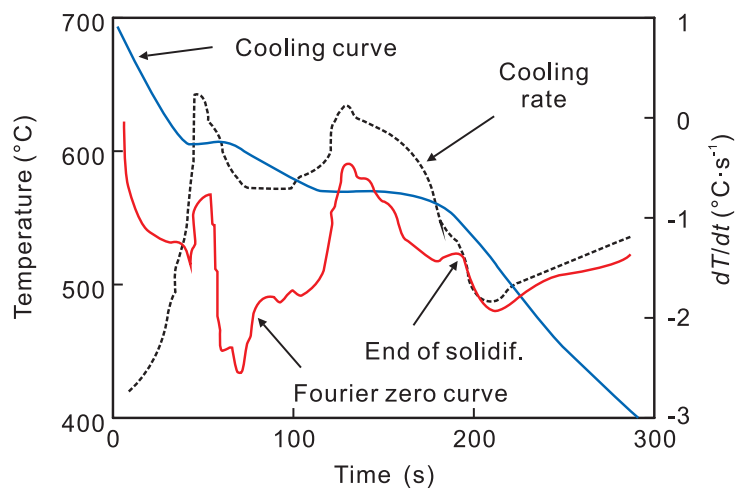

Fig. 9: Cooling curve, cooling rate and Fourier zero curve for an aluminum alloy ${ }^{[24]}$

\section{Applications in cast iron processing}

In industrial practice, a variety of methods and devices are used to extract useful information from the cooling curves of solidifying alloys. The goals of the analysis include estimation of chemical composition, prediction of carbide formation and inoculation efficiency, graphite shape, shrinkage propensity, and mechanical properties.

\subsection{Estimation of chemical analysis}

The first application of TA in process control of cast iron was to evaluate the carbon equivalent (CE). Carbon equivalent is a parameter that considers the fact that cast iron is a multicomponent alloy. It takes into account other important elements
(Si, Mn, P, S, etc.), allowing the multicomponent iron to be treated like a binary Fe-C alloy on the equilibrium diagram. It can be calculated from equilibrium thermodynamics as ${ }^{[26]}$.

$$
\begin{aligned}
& \mathrm{CE}=\% \mathrm{C}_{\text {anal }}+0.31 \cdot \% \mathrm{Si}-0.27 \cdot \% \mathrm{Mn}+0.33 \cdot \% \mathrm{P} \\
& +0.4 \cdot \% \mathrm{~S} \pm m_{X} \% \%
\end{aligned}
$$

where $\% \mathrm{C}_{\text {anal }}$ is the carbon content obtained from chemical analysis, $\% \mathrm{Si}, \% \mathrm{Mn}, \ldots, \% X$ are the other elements' contents, and $m_{X}$ is the coefficient for the various elements (e.g. 0.31 for $\mathrm{Si},-0.27$ for $\mathrm{Mn})^{[27]}$.

However, because solidification is a non-equilibrium process, the thermodynamic equilibrium liquidus temperature is, in most cases, higher than the actual non-equilibrium temperature measured by the thermocouple. The evaluation of $\mathrm{CE}$ through TA is based on the correlation between the non-equilibrium (experimental) liquidus temperature of an alloy $\left(T_{\mathrm{LA}}\right)$ and its chemical composition. Humphreys ${ }^{[28]}$ has introduced the Carbon Equivalent Liquidus (CEL) based on experimental data:

$$
C E L=13.46-0.0081 \cdot T_{\mathrm{L}} \text { where } C E L=\% \mathrm{C}+\% \mathrm{Si} / 4+\% \mathrm{P} / 2
$$

Discussing thermal analysis of SG iron, Sillén ${ }^{[29]}$ argued that the True Eutectic Temperature (TET) is the temperature where the liquidus temperature coincides with the grey eutectic temperature (TE_low) because the carbon equivalent is affected not only by the chemical composition, but also the cooling rate and the nucleation level. He advocates the use of the active carbon equivalent (ACEL), implemented in the commercial TA equipment ATAS. For hypoeutectic ductile iron it is calculated as:

$$
A C E L=14.45-0.0089 \cdot T_{\mathrm{L}}
$$

To determine both $\mathrm{C}$ and Si through TA, Donald and Moore ${ }^{[30]}$ used two cups, a standard one and one that had some tellurium addition. The method requires the use of a correction factor for Si that depends on the phosphorus content of the iron, which makes it cumbersome. The commercial equipment by Heraeus Electro-Nite ${ }^{[31]}$ uses Eq. 5 and the two-cup system. In addition, for ductile iron, a three-cup system is offered to determine the magnesium content. The system includes a standard cup, one with tellurium, and one with tellurium and sulfur.

Standard methods for chemical analysis evaluation of cast iron provide the total, or "residual", $\mathrm{Mg}$ content, i.e., free $\mathrm{Mg}$ plus $\mathrm{Mg}$ bound in compounds such as $\mathrm{MgS}, \mathrm{MgO}$, and silicates. Yet, what controls the graphite shape is the free $\mathrm{Mg}$ termed "active Mg". Suarez et al. ${ }^{[32]}$ developed a method of $\mathrm{Mg}$ estimation by thermal analysis, which consists of the addition of a well-defined amount of $\mathrm{Mg}$ neutralizer mixture $(22 \% \mathrm{~S}$ and $78 \%$ inoculant) in a Te-containing cup. While Te promotes white iron when gray iron is poured in a Te-containing cup (Fig. 10), no significant undercooling is observed with SG iron, probably because of a $\mathrm{Mg}$-Te reaction. When the Mg neutralizer mixture is added into the Tecontaining cup, $\mathrm{Mg}$ is preferably reacting with sulfur. Then, if the resulting cooling curve indicates white iron, it means that the free $\mathrm{Mg}$ in the melt has combined with $\mathrm{S}$, and therefore Te has produced white iron. On the contrary, if the cooling curve corresponds to a graphitic iron, it means that there is some 


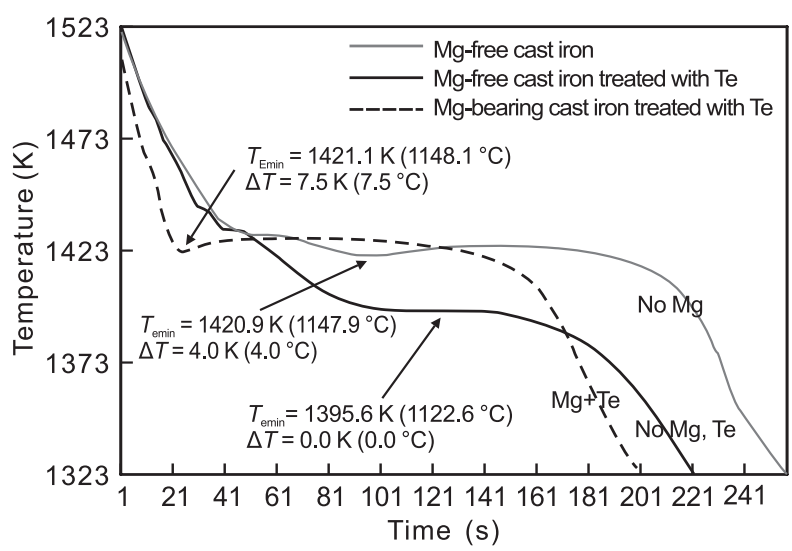

Fig. 10: Cooling curves recorded with different cast iron samples ${ }^{[32]}$

unreacted $\mathrm{Mg}$, so no carbide-stabilizing effect of tellurium is observed. The minimum active $\mathrm{Mg}$ content in the sample to stop the whitening effect of Te mixture was established to be $0.006 \%$. This procedure does not provide the amount of active $\mathrm{Mg}$. It is used as a threshold to determine if the $\mathrm{Mg}$ is above a certain limit ${ }^{[7]}$. This threshold is given by the $\% \mathrm{~S}$ added, which can be varied depending on the casting size and the production process.

\subsection{Prediction of graphitization potential}

Because the cooling curve is sensitive to the amount of carbides that solidify (Fig. 11), it can be used to predict the type of solidification, i.e. gray (stable) or white (metastable). This evaluation is possible if the stable $T_{\text {st }}$ and metastable $T_{\text {met }}$ temperatures can be calculated or measured. The principle is described in Fig. 12. If both the start and end of the eutectic solidification are above the metastable temperature, $T_{\text {met }}$, the iron solidifies gray, i.e. without carbides (Fig. 12a). If the end of the solidification is under $T_{\text {met }}$ the iron is mottled (mixed gray and white Fig. 12b). If both the beginning and end of solidification are under $T_{\text {met }}$, the iron is white (Fig. 12c). The $T_{\mathrm{st}}$ and $T_{\text {met }}$ are not straight line, as they are affected by microsegregation during solidification. Carbide promoting elements are rejected in the liquid, their content increases, and $T_{\text {st }}$ bends downward, while $T_{\text {met }}$ bends upward. The opposite is true for graphite promoting elements.

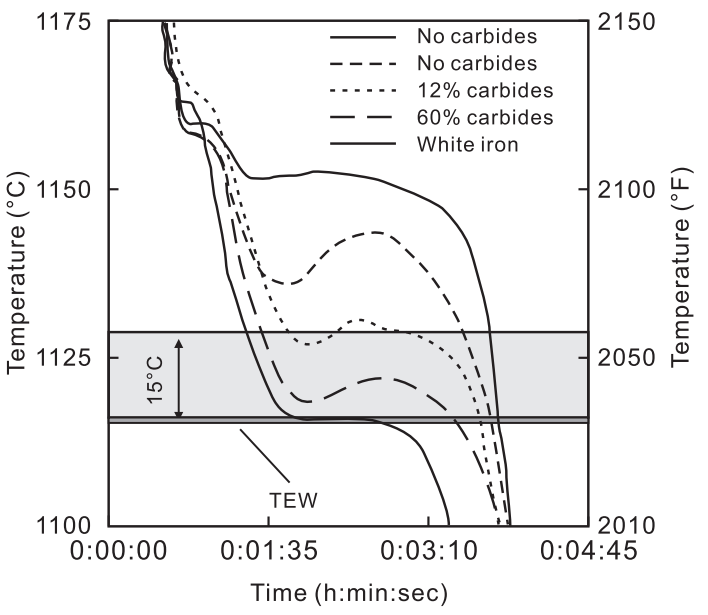

Fig. 11: Effect of amount of carbides on cooling curve ${ }^{[7]}$
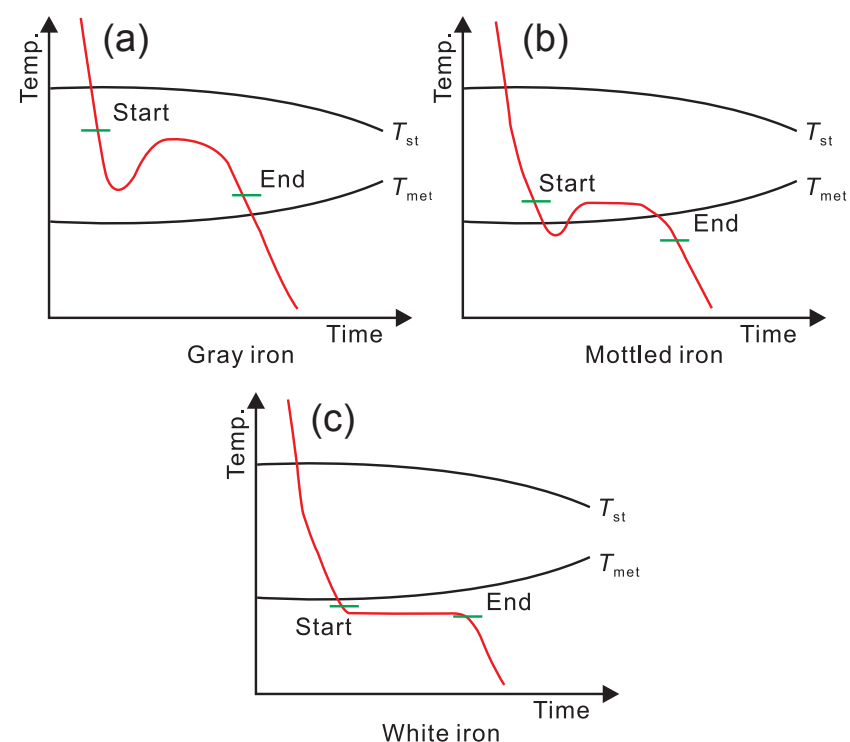

Fig. 12: Interpretation of cooling curves to predict stable or metastable solidification in cast iron ${ }^{[8]}$

As discussed, the equilibrium $T_{\mathrm{st}}$ and $T_{\text {met }}$ calculated from equilibrium thermodynamics ignore the effect of processing variables such as pouring temperature and inoculation. Kanno et al. ${ }^{[33,34]}$ developed the corresponding equations from experiments:

$$
\begin{aligned}
T_{\mathrm{st}}= & 1149.1+4.7 \cdot \% \mathrm{Si}-4 \cdot \% \mathrm{Mn}-44 \cdot \% \mathrm{P}+2.7 \cdot \% \mathrm{Cu}+\% \mathrm{Ni} \\
& +1.8 \cdot \% \mathrm{Co}+13.9 \cdot \% \mathrm{Al}-17.7 \cdot \% \mathrm{Mo}-10.5 \cdot \% \mathrm{Cr}-9.3 \cdot \% \mathrm{Sn} \\
& -5.2 \cdot \% \mathrm{Sb}-6.1 \cdot \% \mathrm{~W}-3.7 \cdot \% \mathrm{Nb}-14.8 \cdot \% \mathrm{~V}-80.3 \cdot \% \mathrm{~B} \\
T_{\text {met }}= & 1142.6-11.6 \cdot \% \mathrm{Si}-0.75 \cdot \% \mathrm{Mn}-46.2 \cdot \% \mathrm{P}-1.4 \cdot \% \mathrm{Cu} \\
& -1.1 \cdot \% \mathrm{Ni}-0.7 \cdot \% \mathrm{Co}-1.8 \cdot \% \mathrm{Al}-14.5 \cdot \% \mathrm{Mo}+5.9 \cdot \% \mathrm{Cr} \\
& -6 \cdot \% \mathrm{Sn}-5.1 \cdot \% \mathrm{Sb}-2.8 \cdot \% \mathrm{~W}+3.3 \cdot \% \mathrm{~V}-26 \cdot \% \mathrm{~B}
\end{aligned}
$$

where the temperature is in ${ }^{\circ} \mathrm{C}$ and the elements are in mass $\%$. For the case of $\mathrm{Mn}$ and $\mathrm{S}, \mathrm{Mn}$ bonds $\mathrm{S}$ to make $\mathrm{MnS}$, which does not affect the equilibrium temperatures. Therefore, soluble $\mathrm{Mn}$ and soluble S should be calculated and used. The calculation method for soluble $\mathrm{Mn}$ and $\mathrm{S}$ in gray iron was reported by the same researchers ${ }^{[35]}$.

Sparkman and Bhaskaran ${ }^{[36]}$ found a linear relationship between the chill depth of a wedge test and $T_{\mathrm{LA}}$ and $T_{\mathrm{Emin}}$ obtained from a plain cup, and the $T_{\text {Ewhite }}$ obtained from a tellurium cup: chill depth $=k_{1} T_{\mathrm{LA}}+k_{2}\left(T_{\text {Emin }}-T_{\text {Ewhite }}\right)+k_{3}$.

Three one-thermocouple cups (base metal, with inoculant and with tellurium) were used by Kanno et al. ${ }^{[37]}$ to predict the eutectic graphitization ability of gray iron, the quality of the molten metal and even the tensile strength. Using the definitions in Fig. 13, the eutectic graphitization ability, EGA, is calculated as:

$$
E G A=\frac{\mathrm{DT} 1}{\mathrm{DTE}} \cdot 100=\frac{T_{\mathrm{Emin}}(\text { base iron })-T_{\mathrm{E} \min }(\mathrm{Te} \text { cup })}{T_{\mathrm{Emax}}(\text { inoc. iron })-T_{\mathrm{E} \min }(\mathrm{Te} \text { cup })} \cdot 100
$$

The chilling depth, $D$ in $\mathrm{mm}$, for gray iron with the composition in the range $2.9 \%-3.5 \% \mathrm{C}, 1.4 \%-2.4 \% \mathrm{Si}$ and $0.1 \%-1.4 \% \mathrm{Cr}$, is calculated as a function of $E G A$ as follows:

$$
D=-0.27 \cdot E G A+28
$$




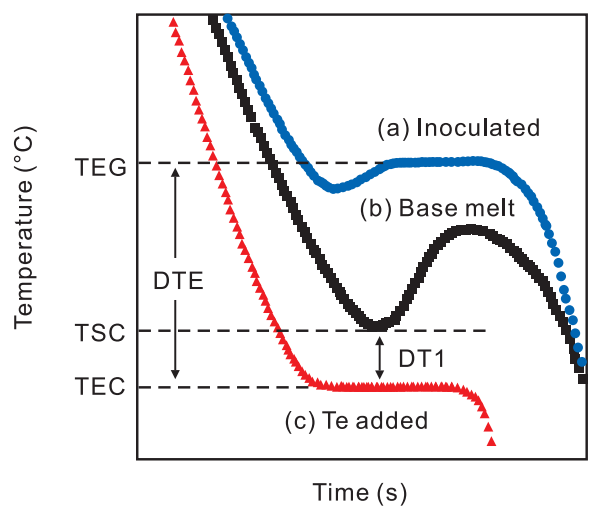

Fig. 13: Definition of terminology used in three-thermocouple thermal analysis of gray iron ${ }^{[37]}$

The three-cups thermal analysis can predict the melt quality for gray iron, but it cannot be used in SG iron because, as the austenite solidifies ahead of graphite, $T_{\text {st }}$ cannot be calculated. In addition, as $\mathrm{Te}$ combines with $\mathrm{Mg}$, it cannot produce chill and $T_{\text {met }}$ cannot be calculated. However, as $T_{\text {st }}$ and $T_{\text {met }}$ can be calculated with Eq. 6 and Eq. 7, Kano et al. ${ }^{[34]}$ developed a one cup method that uses the $T_{\text {Emin }}$ (TSC) from the cooling curve. As the sulfur combines with $\mathrm{Mg}$, it is ignored in the calculation of $T_{\text {st }}$ and $T_{\text {met }}$. Then, EGA can be calculated as:

$$
E G A=\frac{T_{\mathrm{Emin}}-T_{\text {met }}}{T_{\text {st }}-T_{\text {met }}} \cdot 100
$$

\subsection{Prediction of solidification and room- temperature microstructure}

Early work by Ekpoom and Heine ${ }^{[38]}$ demonstrated the existence of a linear relationship between the temperature of the liquidus arrest, $T_{\mathrm{LA}}$, of gray iron and the amount of primary austenite (Fig. 14):

$$
\text { \%austenite }=0.304 T_{\mathrm{LA}}-653.61
$$

Differential thermal analysis as explained in Fig. 6 allows calculation of the amount of primary (austenite or graphite) and eutectic phases produced during the solidification of cast iron ${ }^{[19]}$. A more recent example of calculations and experimental validation is provided in Fig. 15. Note that the start and end of the eutectic solidification were found from the 2nd derivative of the cooling curve.

(a)

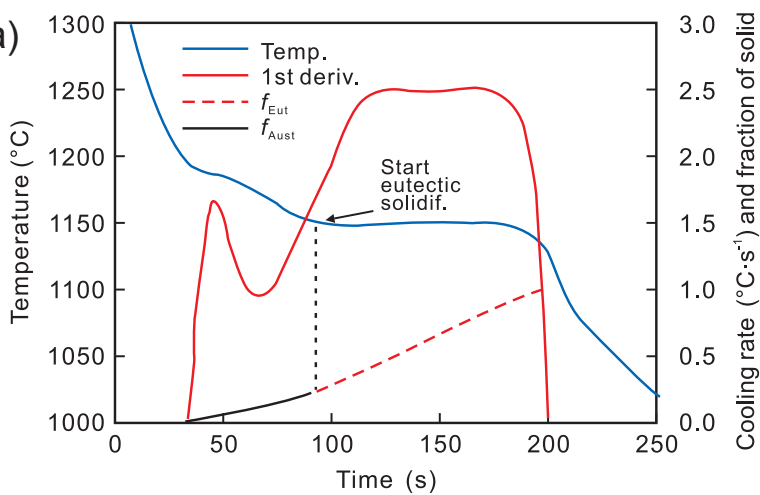

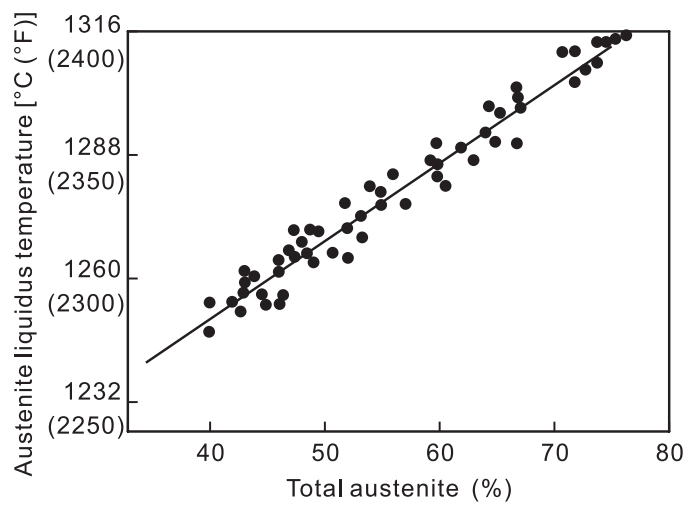

Fig. 14: Relationship between the temperature of liquidus arrest and the amount of primary austenite ${ }^{[38]}$

An important event during solidification is the occurrence of dendrite coherency, which is the time when the individual dendrites first impinge on one another. Before dendrite coherency, flow is unrestricted, as dendrites flow with the liquid. After dendrite coherency, flow is restricted by the solid, rigid network of dendrites, and feeding of the casting is constrained. Anjos et al. ${ }^{[10]}$ used the dual-cup system presented in Fig. 2 to determine the time of dendrite coherency during solidification. According to Bäckerud et al. ${ }^{[39]}$ the curve representing the temperature difference between the solidification curve in the center and that in the wall can be used to find the coherency point; it will correspond to the first minimum in the temperature difference curve occurring after the start of solidification, as shown in Fig. 16. A projection of this point on the $1 \mathrm{st}$ and 2nd derivative curves (bottom of Fig. 16) shows that it approximately matches the maximum of the 1st derivative curve and the zero of the 2 nd derivative of the temperature-time curve of the thermocouple in the center. Thus, the maximum cooling rate after the liquidus indicates the time at which the dendrite coherency occurs.

TA has also been used for a number of researchers to attempt prediction of room temperature microstructure. Guo and Stefanescu ${ }^{[40]}$ studied the effect of cooling rate during the eutectoid transformation on the microstructure of ferriticpearlitic $(0.19 \% \mathrm{Mn}, 0.13 \% \mathrm{Cu})$ and pearlitic $(0.31 \% \mathrm{Mn}, 0.28 \%$ $\mathrm{Cu}$ ) SG irons. They demonstrated that, for the ferritic sample, at a relatively low cooling rate of $0.2 \mathrm{~K} \cdot \mathrm{s}^{-1}$, the 1 st derivative detects two apexes (Points 2 and 4 on Fig. 17a), corresponding to the

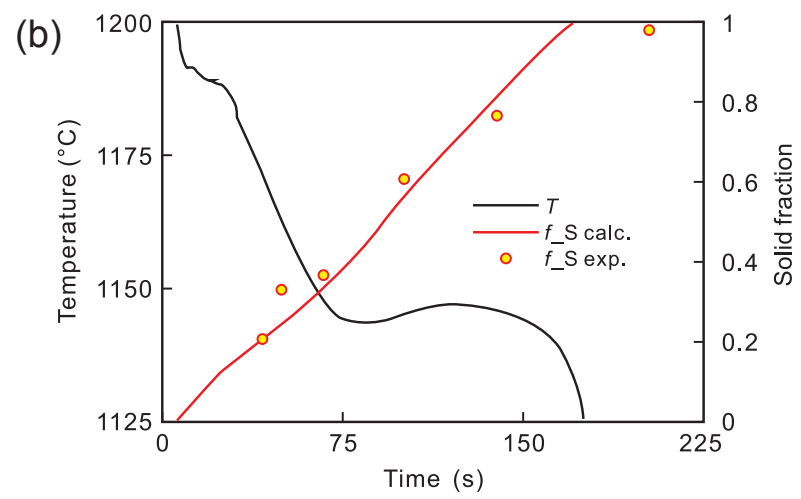

Fig. 15: Calculation of solid fraction evolution for primary austenite and eutectic: (a) comparison with experimental results obtained through quenching experiments; (b) adapted after Ref. [22] 
(a)

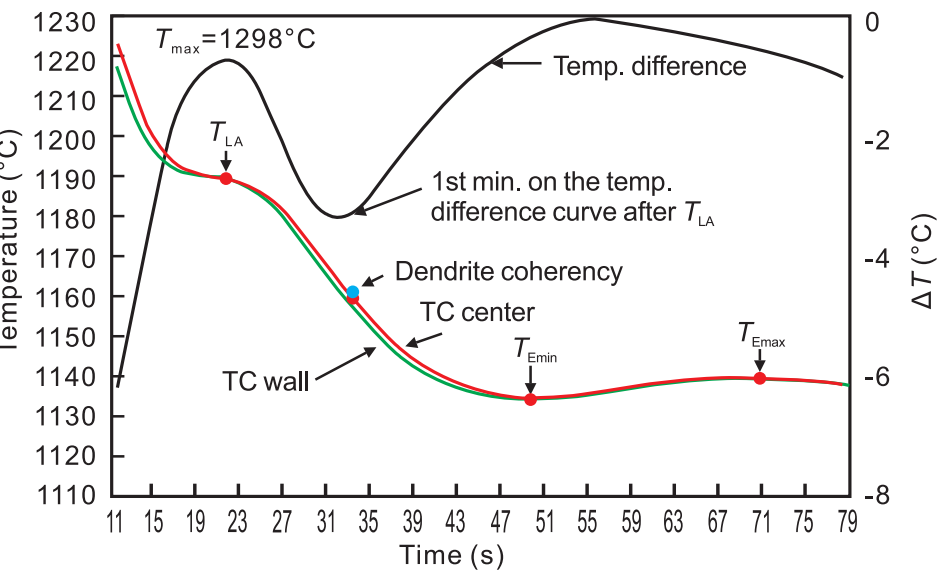

(b)

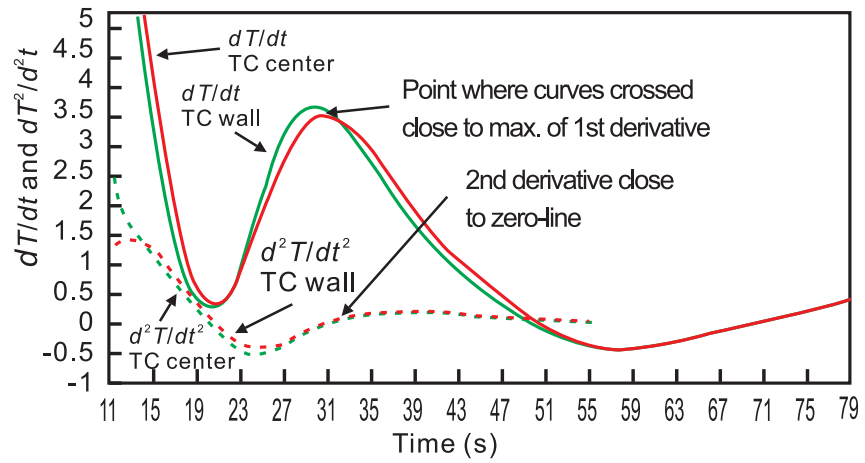

ferrite and pearlite transformation. Only one apex is seen for a higher cooling rate of $0.4 \mathrm{~K} \cdot \mathrm{s}^{-1}$, which indicates that only a pearlitic transformation occurs (Fig. 17b).

In a more recent study, Sertucha et al. ${ }^{[4]}$ studied the effect of inoculation and changes in the $\mathrm{Cu}$ and $\mathrm{Mn}$ content on the room temperature microstructure of a large number of industrial SG irons cooled with $\sim 50 \mathrm{~K} \cdot \mathrm{min}^{-1}$. The main cooling curve parameters included in their analysis are shown in Fig. 18. Note the velocity of transformation $V_{\text {trans }}$ was measured at the point of inflection of the eutectoid transformation, and $T_{\text {trans }}$ corresponding to this point was calculated from the maximum of the 1st derivative.

Some reasonable correlations were found between the measured amount of ferrite and the parameters $V_{\text {trans }}$ and $T_{\text {trans }}$, as shown in Fig. 19.

A statistical analysis equation was derived from the experimental data as a function of cooling curve parameters: \%ferrite $=f\left(T_{\mathrm{Emin}}, \Delta T, V_{\mathrm{Emax}}, V_{\text {trans }}, I_{\mathrm{Gr}}\right)$, where $V_{\text {Emax }}$ is the maximum cooling rate at the final step of the eutectic transformation, and $I_{\mathrm{Gr}}$ is the

Fig. 16: Determination of the dendritic coherency point ${ }^{[10]}$
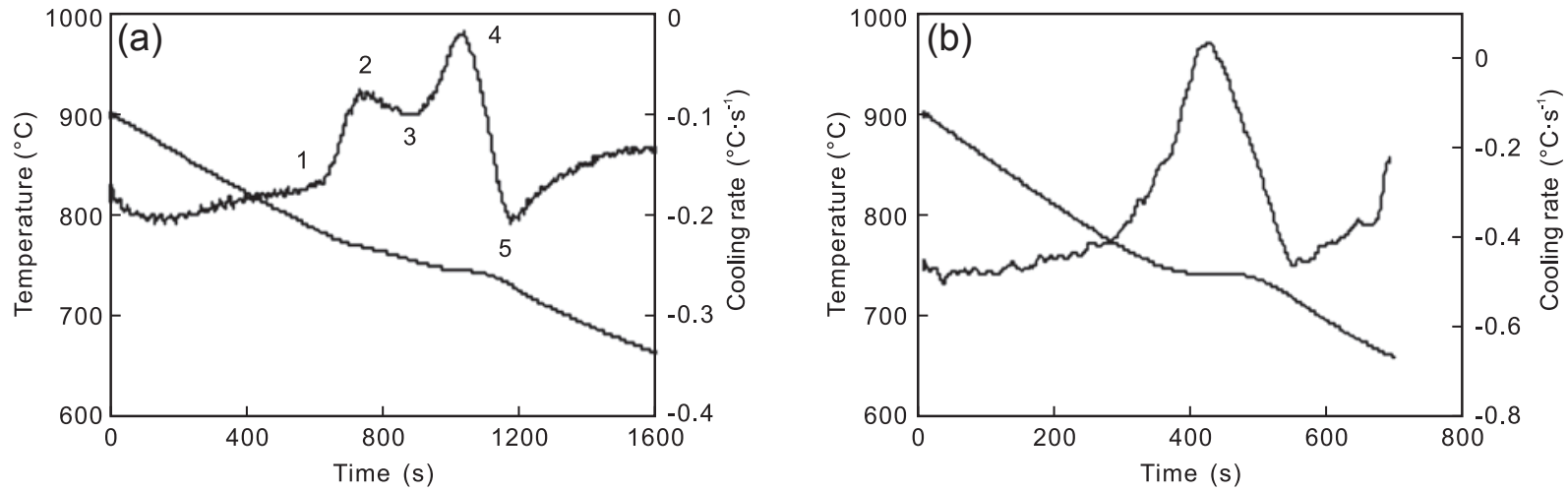

Fig. 17: Cooling curves and their first derivative curves for ferritic SG iron: (a) $0.2 \mathrm{~K} \cdot \mathrm{s}^{-1}$ cooling rate; (b) $0.4 \mathrm{~K} \cdot \mathrm{s}^{-1}$ cooling rate ${ }^{[40]}$

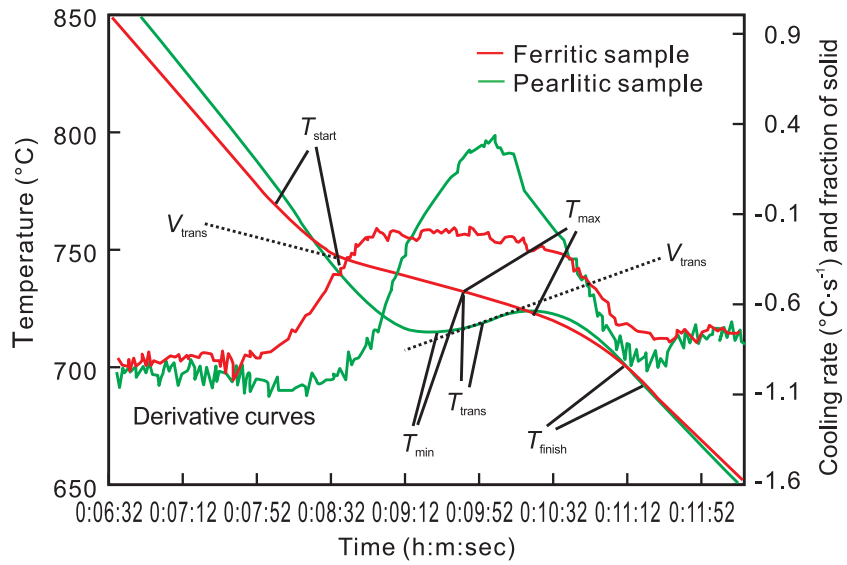

Fig. 18: Critical parameters of the cooling curve for the region of solid-state transformation ${ }^{[41]}$ graphite growth factor at the eutectic calculated as:

$$
I_{\mathrm{Gr}}=\frac{M_{\text {sample }}\left[\% \mathrm{C}_{\text {sample }}-\left(2.03-0.2 \% \mathrm{Si}_{\text {sample }}\right)\right]}{100 t_{\mathrm{Gr}}}
$$

Here, $M_{\text {sample }}$ is the mass of the sample, $t_{\mathrm{Gr}}$ is the graphite growth time in the eutectic range. A good agreement was found between the calculated ferrite content and the experimental measurements on the metallographic samples.

\subsection{Prediction of inoculation efficiency and nodule count}

Inoculation is an important part of the processing of LG and SG irons. Inoculation is a transitory process and its effect decreases with the holding time (Fig. 20), a process called fading. Thus, prediction of inoculation efficiency 
(a)

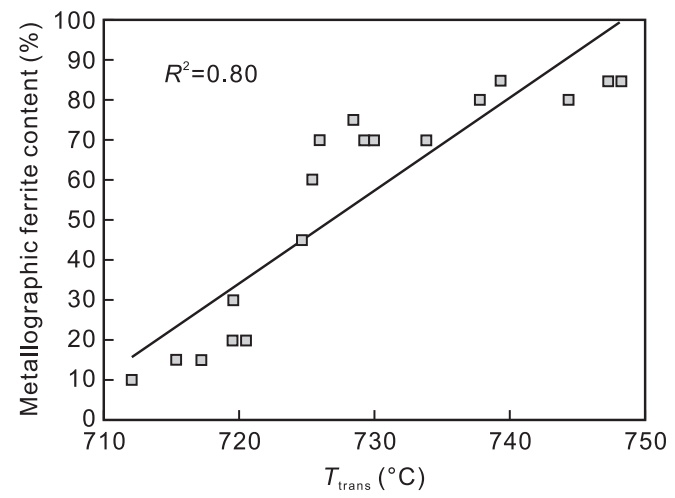

(b)

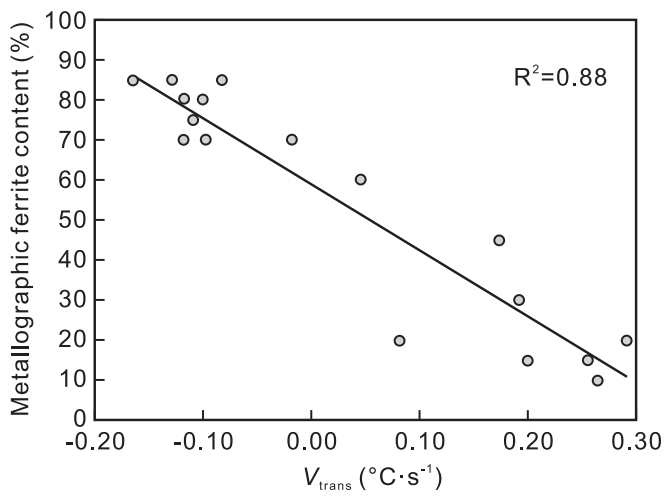

Fig. 19: Correlation between parameters of cooling curve and \% ferrite measured on metallographic samples ${ }^{[41]}$

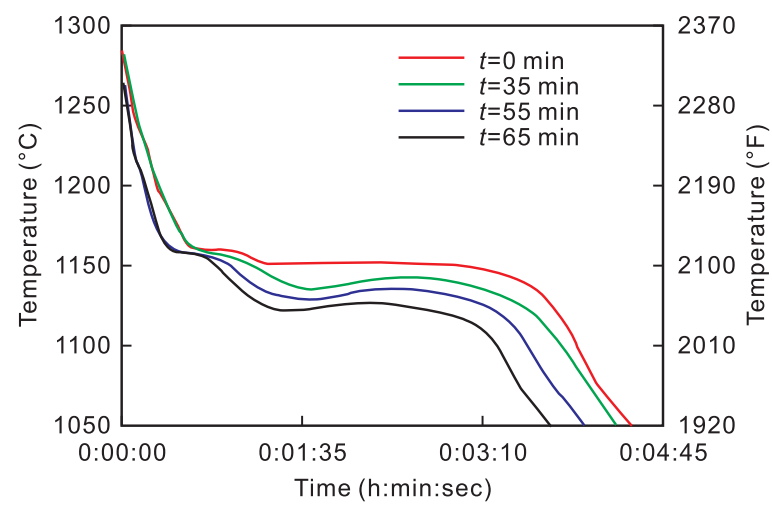

Fig. 20: Effect of inoculation fading on the cooling curves of SG iron ${ }^{[7]}$

is a significant part of process control. Various inoculants will produce cooling curves with different undercooling. In principle, the best inoculant generates the lowest undercooling.

For SG iron, inoculation efficiency is measured by the ability of the inoculant to prevent carbide formation and to insure a large number of SG aggregates (high nodule count). It can be estimated using the eutectic graphitization ability, EGA Eq. 10, as shown in Fig. 21. Based on this figure, the nodule count can be calculated as:

$$
N=6.13 \times 10^{2} \times \frac{T_{\text {Emin }}-T_{\text {met }}}{T_{\text {st }}-T_{\text {met }}}-126
$$

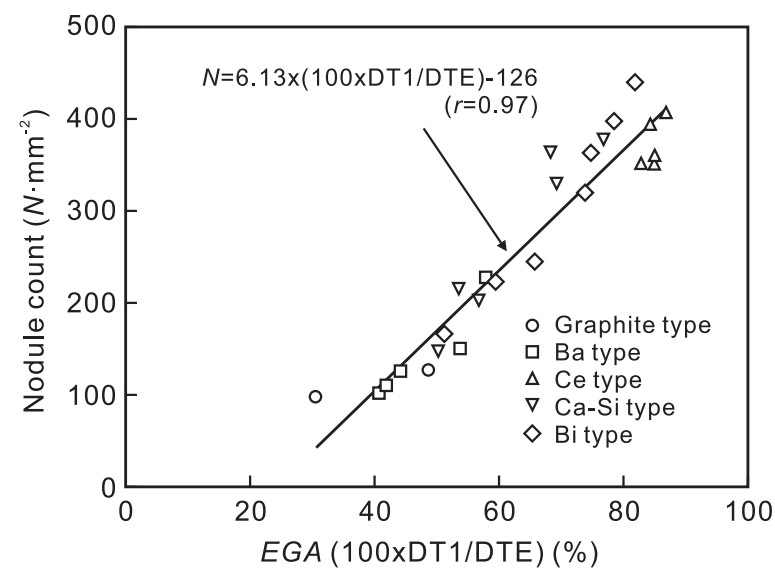

Fig. 21: Relationship between EGA and the graphite nodule count for irons inoculated with various alloys ${ }^{[34]}$
Another nodule count prediction method relies on an equation developed from the statistical analysis of experimental data ${ }^{[42]}$. The nodule count, $N$, is a function of selected parameters obtained from TA: $N=f\left(T_{\text {Emin }}, \Delta T,(d T / d t)_{\max }, G_{\mathrm{R}}\right)$. The TA notations are those listed in Table 1, and $G_{\mathrm{R}}=\sum m_{\mathrm{Gi}} /\left(\Delta t_{\mathrm{i}}\right)$, a parameter defined from the shape of the cooling curve, is the graphite formation rate calculated from the mass of graphite precipitated $m_{\mathrm{Gi}}$ at each time step $\Delta t_{\mathrm{i}}$.

\subsection{Prediction of graphite shape}

The graphite shape can be estimated from information extracted from the cooling curve at the end of solidification, as shown in Fig. 22 for Mg-treated irons with $\mathrm{Mg}$ contents of $0.006 \%$ to $0.031 \%{ }^{[13]}$. A good correlation of 0.93 was found between nodularity and the time interval at the end of eutectic reaction (on 2 nd derivative) $\Delta t_{\text {end. }}$. However, data from similar heats but also containing $\mathrm{Ti}$, lined up above the regression line in the figure.

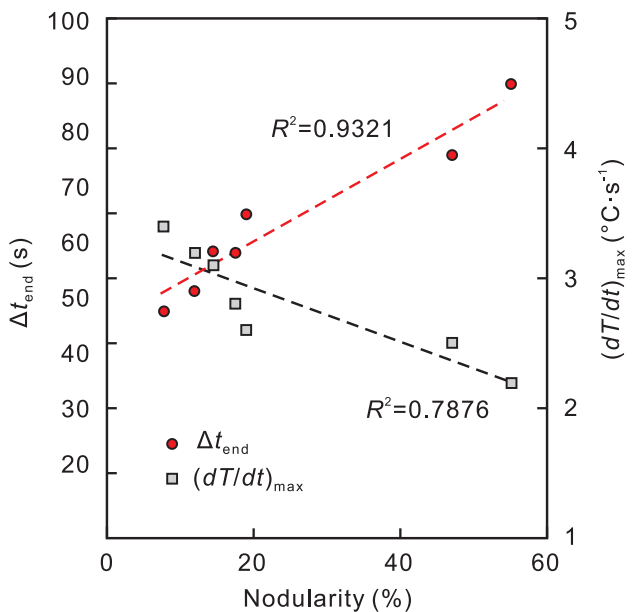

Fig. 22: Estimation of nodularity using information from the cooling curve at the end of solidification $\left(\Delta t_{\text {end }}\right.$, and $(d T / d t)_{\max }$, the maximum cooling rate at the end of solidification) ${ }^{[13]}$

Some thermal analysis systems relate the angle $\theta$ on the first derivative of the cooling curve at the end of the solidification (also labeled as GRF2 by some systems) with the shape of the graphite (Fig. 23). Low angles correspond to high thermal conductivity. Gray irons typically show lower angles than ductile irons. 


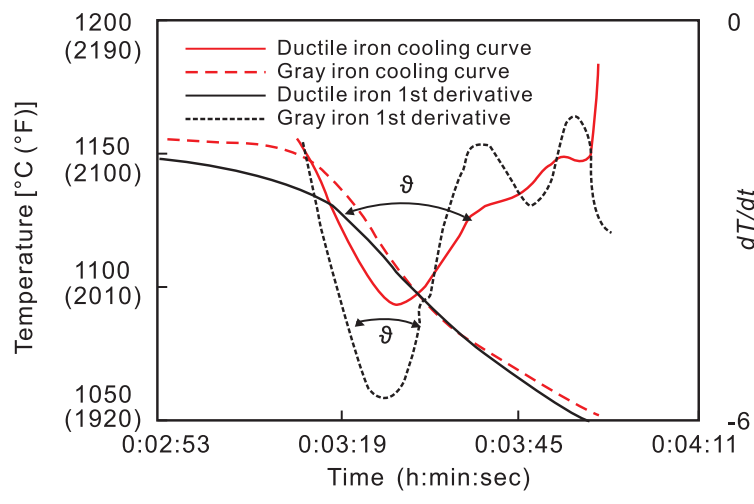

Fig. 23: Comparison of end of solidification for gray and ductile iron ${ }^{[7]}$

Compacted graphite iron presents a particularly difficult processing case as the magnesium window is very narrow. This is because the graphite shape is also affected by the cooling rate and inoculation that enhance graphite spheroidization. Perhaps the best example of successful application of thermal analysis in the process control of cast iron is in the production of CG iron by Sintercast, the most widely used method in the industry for production of CG iron ${ }^{[43]}$. The uniqueness of the process that uses a one-cup made of steel consists in the intentional undertreatment of the iron followed by ladle correction. The system is based on the concept of on-line Measure-and-Correct that in summary includes the following steps: (1) the liquid iron is undertreated with a Mg-containing alloy; (2) a steel thermal analysis cup is poured at a closely controlled temperature; (3) depending on the result of the TA, a wire feeder inserts into the pouring ladle a calculated amount of inoculant and magnesium to bring the iron to the optimum chemical composition for pouring.

An important issue in the production of heavy section ductile iron castings is the avoidance of chunky graphite (CHG). It is currently an active subject of research as evident in some recent reviews ${ }^{[44,45]}$. Attempts have been made to correlate crystallization of chunky graphite with parameters of the cooling curve. Thermal analysis by Sertucha et al. ${ }^{[46]}$ of large blocks (thermal modulus $5 \mathrm{~cm}$ ) produced from SG irons with $4.28 \%$ to $4.38 \% \mathrm{CE}$ that had tendency to generate chunky graphite, exhibited up to three distinct thermal arrests, all at temperatures lower than the stable eutectic temperature, confirming earlier work by Chaudhari et al. ${ }^{[47]}$. Example of the recorded curves with notations as suggested by Chaudhary are presented in Fig. 24. The sequence of solidification as seen on the cooling curve and confirmed from metallographic samples includes nucleation of primary austenite graphite in the liquid $\left(T_{\mathrm{L}}\right)$, initial eutectic reaction consisting of encapsulation of primary nodules by austenite dendrites ( $T_{\mathrm{EN}}$ arrest), initial limited growth of eutectic $\left(T_{\mathrm{EU}}\right)$, and bulk eutectic growth $\left(T_{\mathrm{ER}}\right)$. CHG cells and secondary nodules arranged into SG cells formed during the bulk eutectic growth. Attempts were made to correlate the volume of CHG in the test blocks to information extracted from TA. No consistent correlations were found. The best one appears to be with the recalescence on the TA cups, shown in Fig. 25. Surprisingly, the lowest amount of CHG appears to be in some of the uninoculated samples.

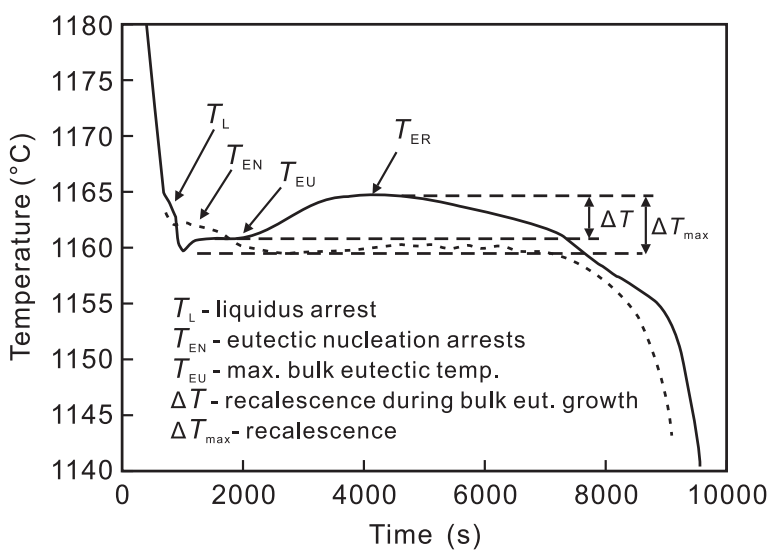

Fig. 24: Cooling curves of blocks with $5 \mathrm{~cm}$ thermal modulus produced from iron with $4.3 \mathrm{CE}$ and $0.42 \% \mathrm{Mg}$ : full line - inoculated; dotted line - not inoculated ${ }^{[46]}$

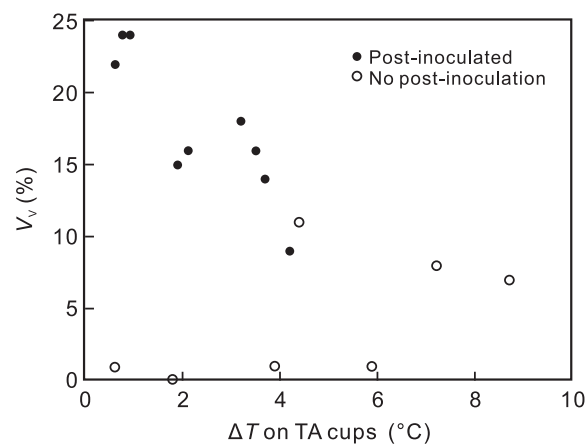

Fig. 25: Correlation between the volume of $\mathrm{CHG}$ regions in the test blocks $\left(V_{\mathrm{v}}\right)$ and $\Delta T_{\max }$ measured from TA on cups ${ }^{[46]}$

Regarding the notation TEN (initial eutectic reaction consisting of encapsulation of primary nodules by austenite dendrites), it is now accepted that for the case of hypereutectic SG iron, this is an austenite liquidus arrest found on the phase diagram when the concept of asymmetric eutectic region is used.

\subsection{Prediction of shrinkage propensity}

After cast iron is poured in a mold and the liquid metal cools, liquid shrinkage occurs as the density of the liquid increases and its specific volume decreases. During solidification, some graphite expansion may compensate the shrinkage. The interplay between these two phenomena produces several shrinkage defects illustrated in Fig. $26^{[48]}$. Some of these defects are the result of uncompensated shrinkage (pipe, caved surfaces), some are produced by a decrease in the gas solubility in the liquid alloy (center-line microporosity), or form the

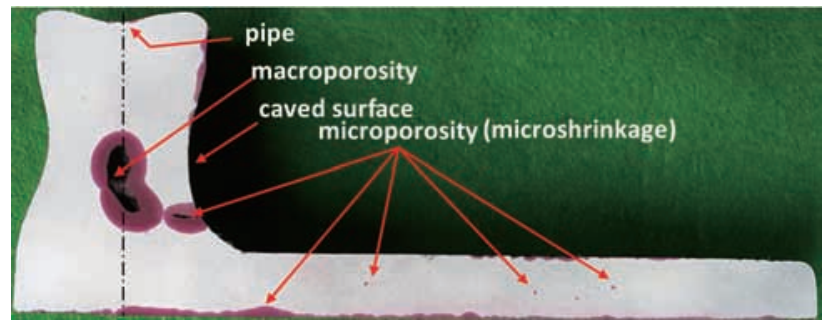

Fig. 26: Various types of shrinkage defects found in an experimental casting ${ }^{[48]}$ 
mold/metal interface interaction (surface microporosity), and some can be the result of both shrinkage and gas porosity (macroporosity or enclosed shrinkage).

During primary solidification of hypoeutectic iron, in the dendrite dominated solidification (Fig. 4), the liquid/austenite mixture continues to shrink as the specific volume decreases (Fig. 27). As long as the permeability of the mushy dendritic region is sufficiently high, the shrinkage is compensated by flow from the risers. Graphite expansion begins once the maximum undercooling, $T E \_l o w$, is reached and may, or may not, continue to the end of solidification. The final result will be expansion if enough graphite expansion occurs, or shrinkage in

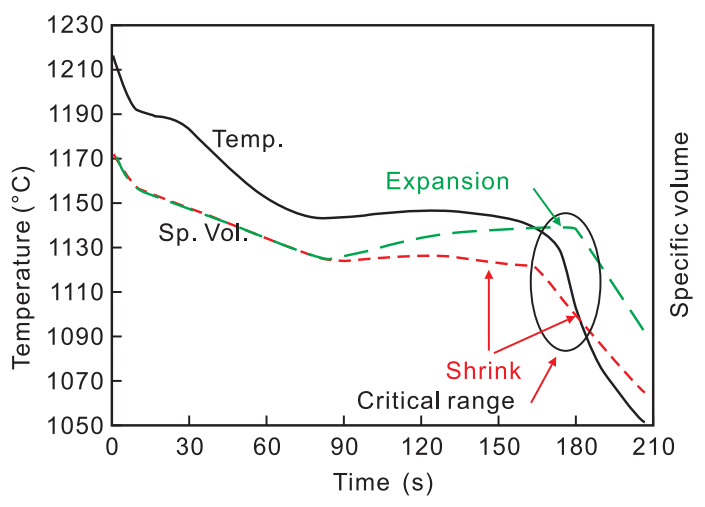

Fig. 27: Temperature and specific volume evolution during the solidification of cast iron the case of insufficient expansion, as shown in the critical range in Fig. 27. The figure also shows a drop in expansion at the end of solidification, typically produced by decreased graphite growth in the last regions to solidify because of segregation of carbides-promoting elements. For SG iron, the permeability of the mushy zone is much lower than that of LG iron. This restricts feeding from the riser and decreases the cooling rate.

In principle, both LG and SG irons of near-eutectic or eutectic composition should exhibit expansion during solidification and therefore should not be prone to forming either cavity or porosity shrinkage. While this is true for gray iron, normal production SG iron is susceptible to shrinkage porosity because of limited graphite growth at the end of solidification, which is then dominated by austenite shrinkage. This induces specific volume decrease and produces uncompensated shrinkage in the last region to solidify, and thus porosity. Mathematically, this is expressed as a negative pressure at the end of solidification, as described in detail in Ref. [48,49]. A bimodal volume size distribution of graphite nodules (small nodules coexisting with large nodules), sometimes observed in SG iron castings, appears to indicate the existence of a second nucleation wave at the end of solidification. This behavior, or extended growth of graphite to the end of solidification, will reduce or even prevent microporosity. It follows that understanding graphite expansion, in particular at the end of solidification, is vital for process development of porosity-free castings.
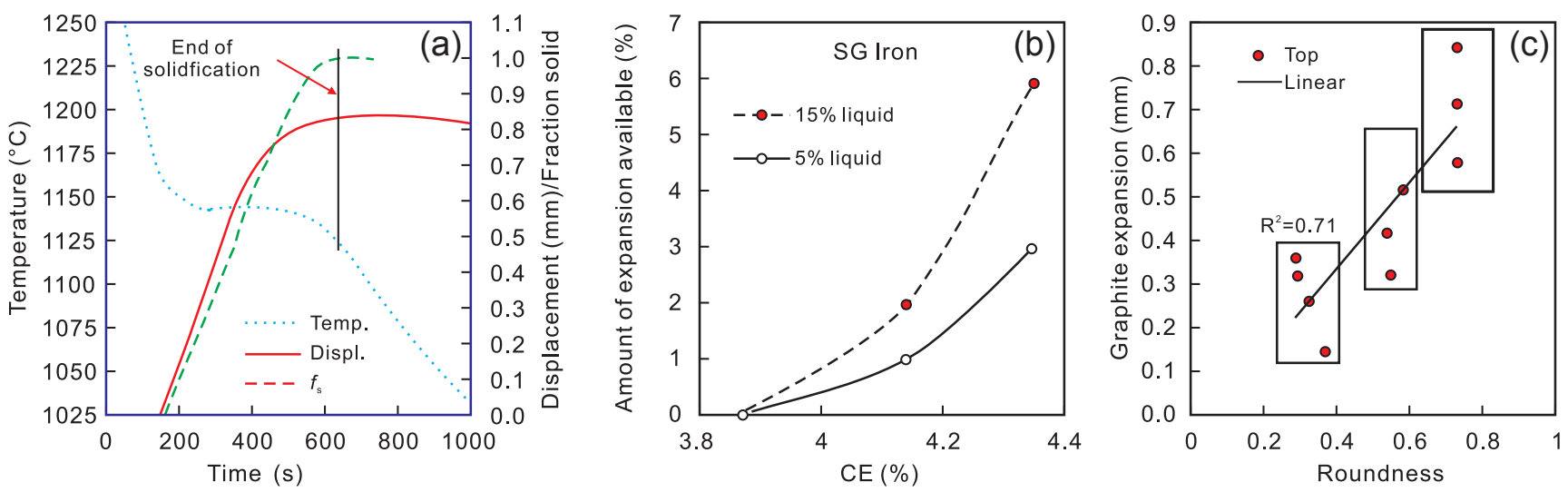

Fig. 28: Interpretation of LDA/TA experiments: (a) time evolution of temperature, linear displacement and fraction solid for a 4.35\% CE SG iron; (b) available graphite expansion at the end of solidification for SG iron; (c) effect of graphite shape on graphite expansion for irons with $4.12 \%$ to $4.44 \% \mathrm{CE}^{[50]}$

To this end, Alonso et al. ${ }^{[50]}$ used a combined Linear Displacement Analysis (LDA) - Thermal Analysis (TA) experimental device to measure the linear displacement during the solidification of cast iron. With this system, it was possible to examine the correlation between temperature, linear displacement, and fraction of solid evolution (Fig. 28a). This in turn made possible calculating the amount of graphite expansion available at the end of solidification which counteracts the austenite shrinkage. Not surprisingly, this expansion increases with $\mathrm{CE}$, as more graphite will be available with higher CE (Fig. $28 b)$. The graphite morphology also has a significant effect. Considerably less graphite expansion of 0.3 to 0.4 was found in CG iron than in SG iron with roundness of 0.7 (Fig. 28c).
From this discussion, it is clear that accurate prediction of shrinkage propensity is highly dependent on accurate evaluation of the permeability of the mushy zone and the pressure change in the zone during solidification. Svidró et al. ${ }^{[49]}$ developed a measurement method and a calculation algorithm to determine the pressure in the mushy zone during solidification. The method involves a spherical sample suspended in a measurement device that records the temperature and the volume changes during solidification. The thermocouples are positioned in the geometrical center of the sphere and at its periphery, to allow for a Fourier analysis of the cooling curves. The calculation algorithm is based on the numerical interpretation of the Clausius-Clapeyron equation where the temperature variation, 
the volume change, and the released latent heat are processed to determine the local pressure during solidification. The cooling curves of an LG iron of $3.85 \%$ CE collected during solidification are presented in Fig. 29 as a function of the fraction of solid. The calculated volume change based on the measured surface displacement is also presented in the figure. It is seen that the sample volume decreases from the start of solidification until the fraction solid reaches 0.32 . The start of the graphite precipitation is also confirmed at $f_{\mathrm{s}}=0.32$ by the temperature minimum on the surface temperature curve $T_{2}$. The maximum volume is reached at the beginning of recalescence. Then, the volume decreases because of insufficient graphite formation to compensate for austenite shrinkage.

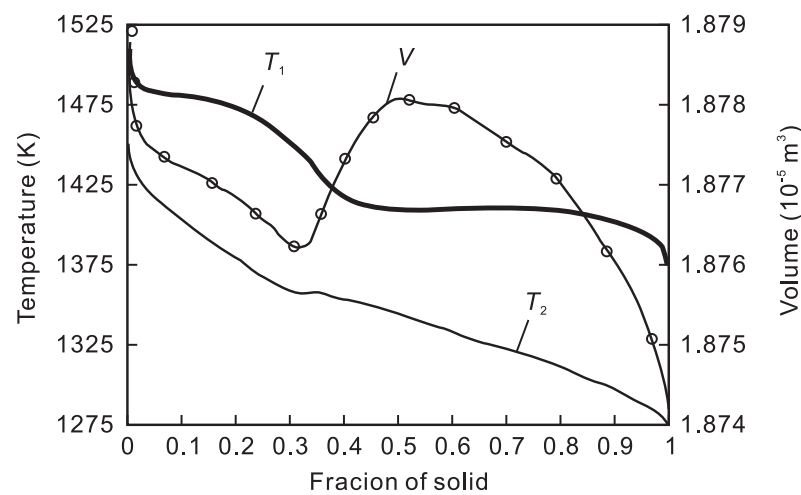

Fig. 29: Cooling curves and calculated volume ( $V$ as a function of fraction solid; $T_{1}$ - cooling curve registered from the geometrical center of the spherical sample, $T_{2}$ - average of the temperatures measured at three different positions on the surface of the sample ${ }^{[49]}$

Figure 30 presents the calculated change in pressure and the temperature in the center as a function of the fraction solid. Positive values on the y-axis are interpreted as compressive pressure, while negative values are considered to be expansion pressure. It is seen that expansion pressure starts at $f_{\mathrm{S}}=0.32$ when the volume begins to increase. At about 0.7 fraction solid, the pressure becomes compressive again and remains so to the end of solidification. Thus, the region $f_{\mathrm{S}}=0.7$ is the region where graphite expansion stops compensating for austenite shrinkage, and microshrinkage may become a problem.

Equally important for producing a shrinkage defect-free casting is the gating-riser system design (accessibility of hot

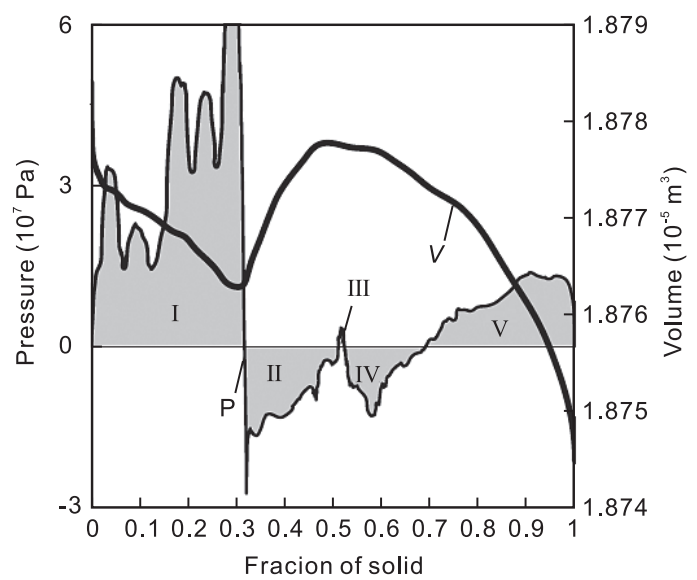

Fig. 30: Calculated pressure change and calculated volume $(V)$ as a function of fraction solid ${ }^{[49]}$

spots by the feeder). If the connection to the riser closes early, piping and caving of the surface can be expected even though graphite expansion at the end of solidification is adequate. In casting production, the problem is further complicated by the mold material (mold rigidity or lack of), pouring temperature, and inoculation.

A study on ductile iron by Stefanescu et al. ${ }^{[51]}$ found the volume of macroporosity decreased and the dispersed microshrinkage increased with higher graphite nodule count. When inoculation was used, despite the decrease in macro-shrinkage, the total dispersed shrinkage increased considerably. Mold rigidity affected the macro-shrinkage but not microporosity formation at the end of solidification. This proved that the graphite nodule count is an important but not sufficient parameter to predict shrinkage porosity in SG iron castings.

The difficulty of predicting shrinkage defect formation can be illustrated using the work of Lekakh and Hrebec ${ }^{[52]}$. Their experimental casting and the measured shrinkage defects are shown in Fig. 31. The effects of inoculation and feeding ability (no riser or different distances from the riser) are illustrated. Different results are seen for the same iron depending on the feeding conditions. Thus, it will be hard to assess shrinkage probability only from a cooling curve information produced with a standard TA system, which can only predict melt quality. Nevertheless, the information that the cooling curve provides on the end of solidification can be used to predict shrinkage
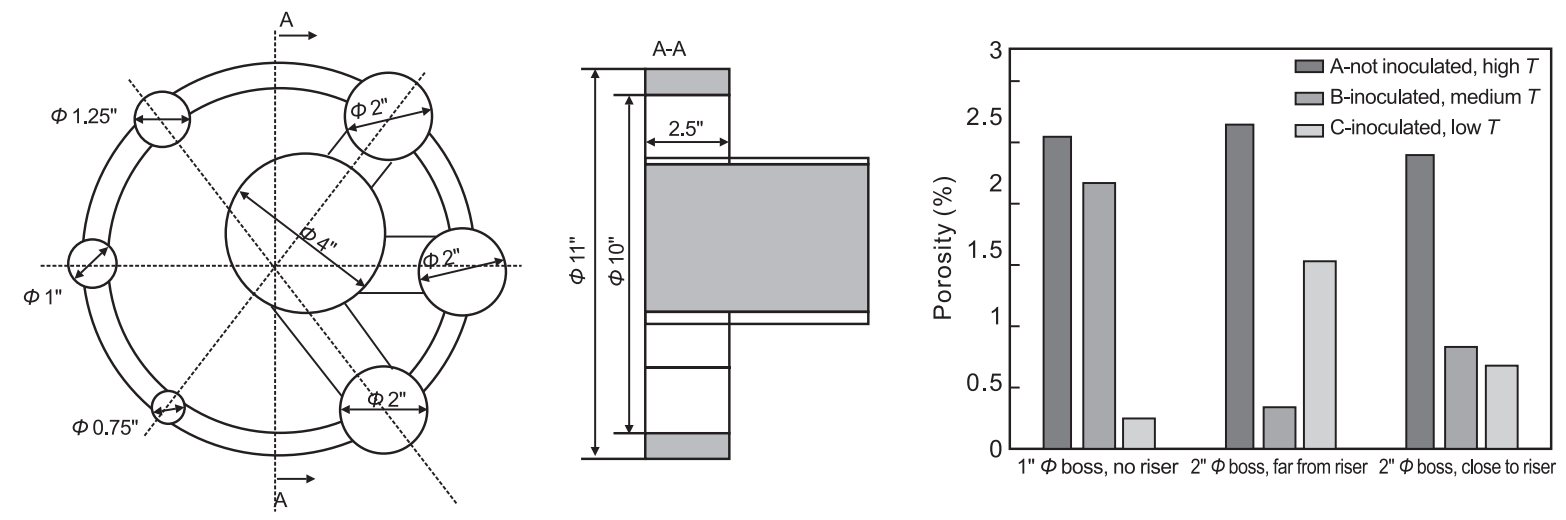

Fig. 31: Experimental casting and measured porosity at different locations in the casting ${ }^{[22]}$ 
propensity of the particular alloy.

Persson and Udroiu ${ }^{[53]}$ indicated that the commercial software ATAS is using the angle $\theta$ formed on the 1st derivative curve after the end of solidification (see $\theta$ in Fig. 23 and GFR 2 in Fig. 32) as a shrinkage tendency indicator. A large $\theta$ angle indicates a high shrinkage tendency. A lamellar graphite iron has a narrower angle at the end of solidification when compared with the spheroidal graphite iron. This is attributed to the differences in the skin-type solidification of LG iron compared to the mushytype solidification of SG iron ${ }^{[8]}$. Very little latent heat is released at the end of solidification of LG iron, which determines a sharp increase in the cooling rate. More latent heat is released at the end of solidification of SG iron as SG grows for a longer time than LG. This produces a slower increase in the cooling rate.

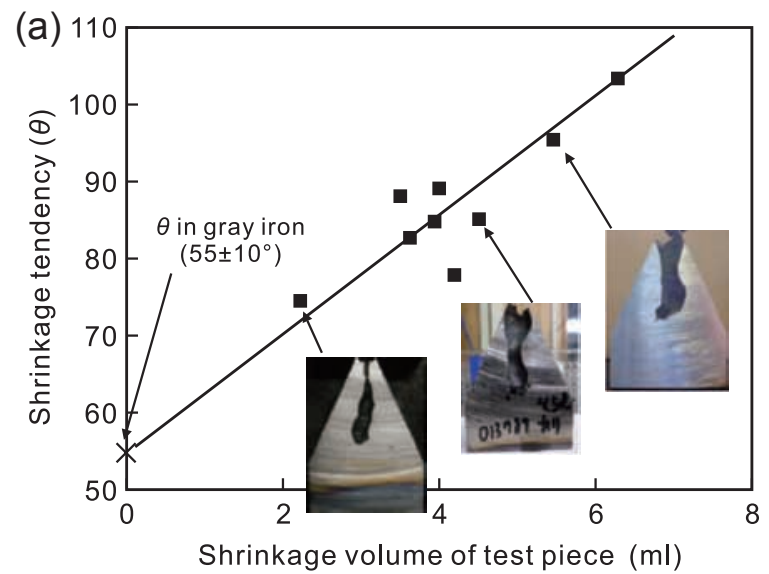

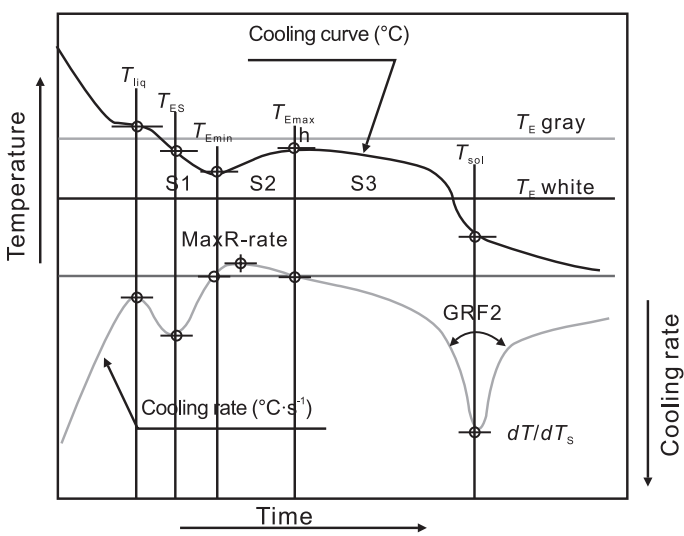

Fig. 32: Parameters of the cooling curve used in analysis by ATAS $^{[33]}$

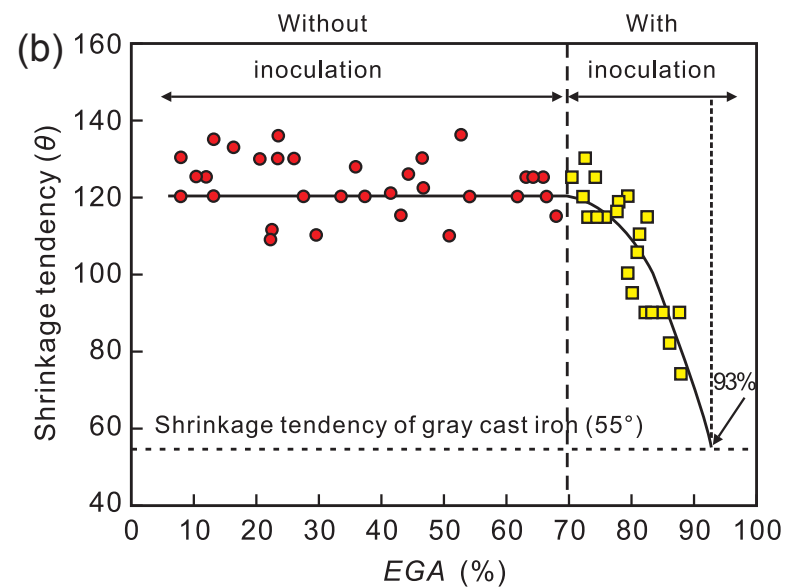

Fig. 33: Correlation between the shrinkage tendency as estimated through the $\theta$ angle on TA with: (a) measured shrinkage volume; (b) nodule count as calculated by $E G A^{[34]}$

Kanno et al ${ }^{[34]}$ used a conical-shape test piece to measure the correlation of shrinkage volume and the $\theta$ angle on the 1-cup TA (Fig. 33a). A good relationship between shrinkage tendency ( $\theta$ angle) and the nodule count as estimated through the EGA Eq. 13 was demonstrated through Fig. 33(b).

Erturk et al. ${ }^{[54]}$ used ATAS to evaluate its applicability to the production of riserless SG iron castings. The experimental variables included inoculation quality, type of resin in the mold, and mold rigidity. According to ATAS instructions, to minimize macro shrinkage, the dendritic dominated zone (1 and 2 in Fig. 4)

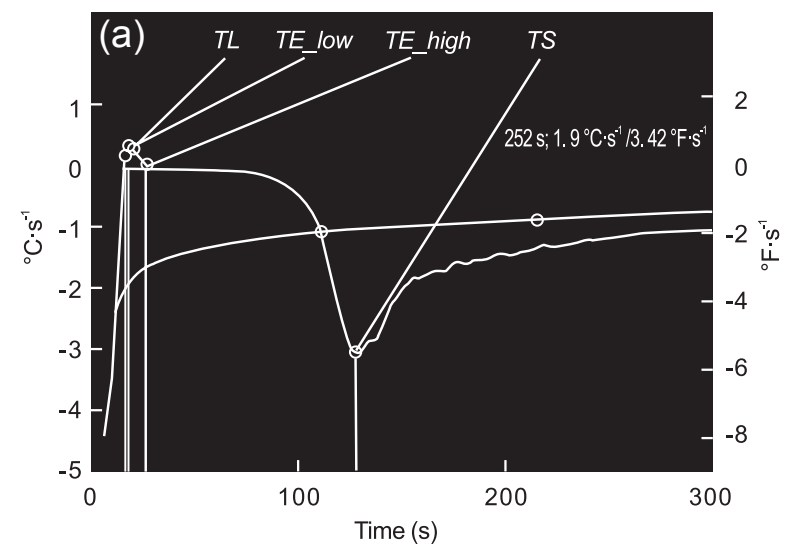

must be as small as possible, and the graphite dominated regions (3 and 4 in Fig. 4), where expansion occurs, must be large. The ATAS thermal analysis system can calculate the free graphite and shrinkage-expansion as a function of temperature from the carbon equivalent (Fig. 34). Using ATAS, the authors were able to calculate the total shrinkage at the end of solidification and design the feeding-casting system for feederless casting.

Another parameter that can be used for shrinkage propensity is the cooling rate at the end of solidification. LG iron has a higher cooling rate at the end of solidification than SG iron.

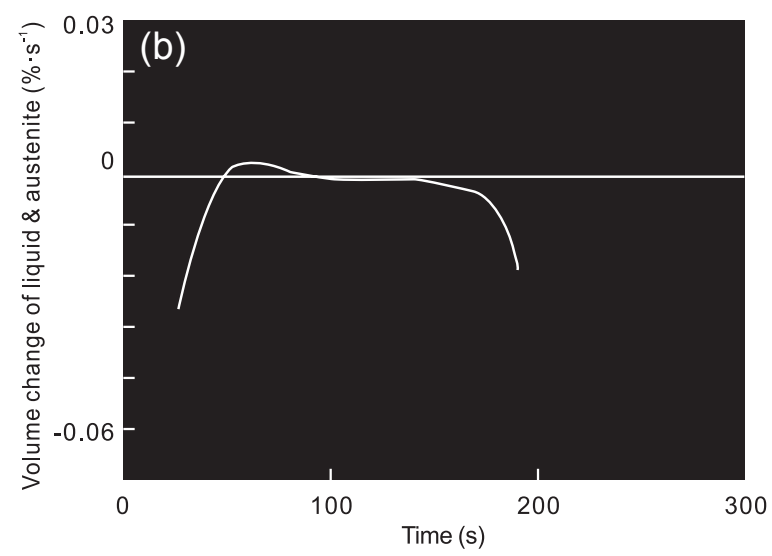

Fig. 34: The first derivative of the cooling curve and the volume change during solidification ${ }^{[54]}$ 
Consequently, the shrinkage tendency can also be estimated through the minimum of the first derivative at the end of solidification ( $d T / d T_{\mathrm{S}}$ in Fig. 32, or ( $\left.d T / d t\right)_{\max }$ in Fig. 5).

Larrañaga et al. ${ }^{[42]}$ used time information from the first derivative of the cooling curve to estimate shrinkage propensity. First, they derived an equation to calculate the volume change between two successive time steps, $v_{\mathrm{i}}$, during solidification:

$$
\Delta v_{\mathrm{i}}=\Delta m_{\gamma}\left(\rho_{\gamma}^{-1}-\rho_{\mathrm{L}}^{-1}\right)+\Delta m_{\mathrm{G}}\left(\rho_{\mathrm{G}}^{-1}-\rho_{\mathrm{L}}^{-1}\right)
$$

where $m$ is the mass, $\rho$ is the density, and the subscripts $\gamma, L$ and $G$ stand for austenite, liquid and graphite, respectively.

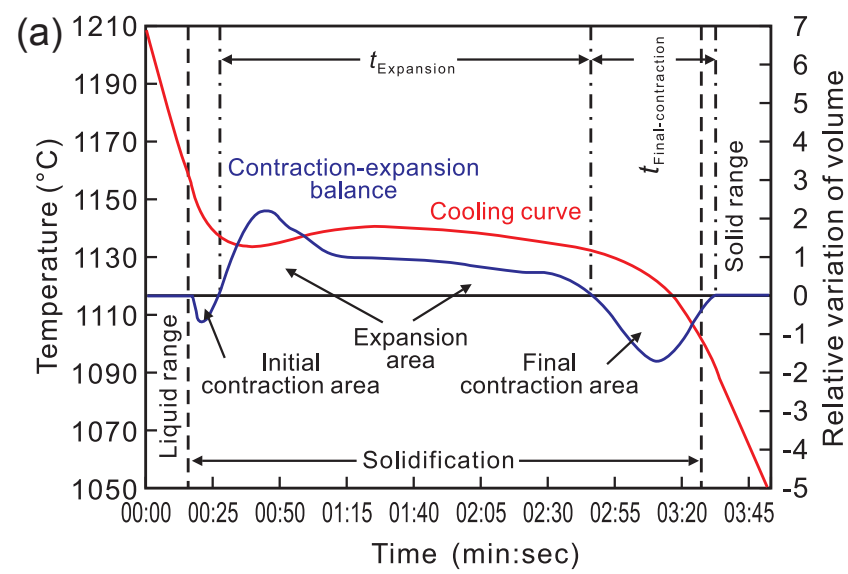

This equation allows the calculation of volume change because of austenite contraction and graphite expansion during solidification, and thus the contraction-expansion balance at different moments during solidification, as shown in Fig. 35(a). They defined the ratio $k=t_{\text {Exp }} /\left(t_{\text {Exp }}+t_{\text {Final_contr }}\right)$ as a predictor for shrinkage porosity in cast iron. A low $k$ is an indicator of high porosity. The model was verified with a cross-shaped casting (poured in the vertical position with a riser on top of the arm of the cross) in conjunction with SG iron. The results are presented in Fig. 35(b). The same authors have also proposed an empirical equation for the calculation of nodule count based on TA data.

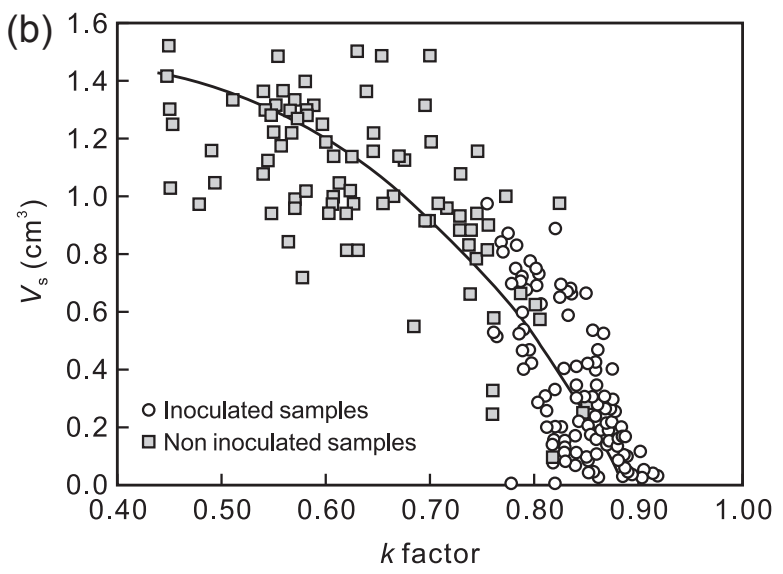

Fig. 35: Use of time-parameters for shrinkage evaluation: (a) suggested correlation between times and areas from the cooling rates curves to microporosity propensity; (b) correlation between the volume of microshrinkage and the $k$ factor ${ }^{[42]}$

A recent novelty in the prediction of microshrinkage propensity is the combination of information obtained from TA with a computer simulation model. Kweon et al. ${ }^{[48]}$ used the fraction solid evolution calculated from the cooling curves recorded on the experimental casting in conjunction with an analytical model. The model captures some of the important elements of the physics of the problem, by calculating gas evolution in the melt with increasing fraction of solid and with decreasing mushy zone permeability during solidification. While calculation results compared well with the experimental observation on the L-shaped castings in Fig. 26, some disagreements were also registered. These were attributed to gas pores migration during solidification, a phenomenon that is not included in the physics described by the model.

\subsection{Prediction of mechanical properties}

The ultimate tensile strength (UTS) and Brinell hardness (HB) in gray iron are directly related to the carbon equivalent, and especially to carbon content, and more generally to eutectic and eutectoid parameters. Because these parameters can be accurately obtained from TA, Udroiu, quoted in Ref. [7], has developed a statistical analysis model for UTS and HB prediction in $30 \mathrm{~mm}$ diameter grey iron test bars based on cooling curves recorded in standard thermal analysis cups. It captures the effect of graphite nucleation and growth, through $T_{\text {Emin }}$ and $\Delta T$, and carbon equivalent $T_{\mathrm{LA}}$, on the final graphite features. The effect of pearlite fineness on the properties of interest is considered through selected features of the eutectoid transformation that include the undercooling and the pearlite growth rate (eutectoid recalescence and maximum cooling rate during the eutectoid transformation). The agreement between predicted and experimental values is shown in Fig. 36.

\section{Summary}

A review of the work performed over the last 90 years for the development of TA and its implementation in industry shows that progress has been made both in the development of the hardware and the software of TA. The consistency of the data provided by the TA depends heavily on two factors: the temperature at which the metal is poured in the cup and the cooling rate (the mass of the metal in the cup) during the test. A higher pouring temperature and a greater mass determine a longer solidification time which affects significantly the undercooling. These are both difficult to control with the one-cup system. To the best of our knowledge, at this time, only two systems satisfy the requirement for constant mass: Sintercast (Fig. 1b) and GmbH (Fig. 2). Only Sintercast satisfies both requirements.

The progress in the interpretation of cooling curves includes correcting the misconception that the maximum cooling rate (min. of $1 \mathrm{st}$ derivative) after $T_{\mathrm{LA}}$ shows the time of the beginning of solidification. It was demonstrated that this 

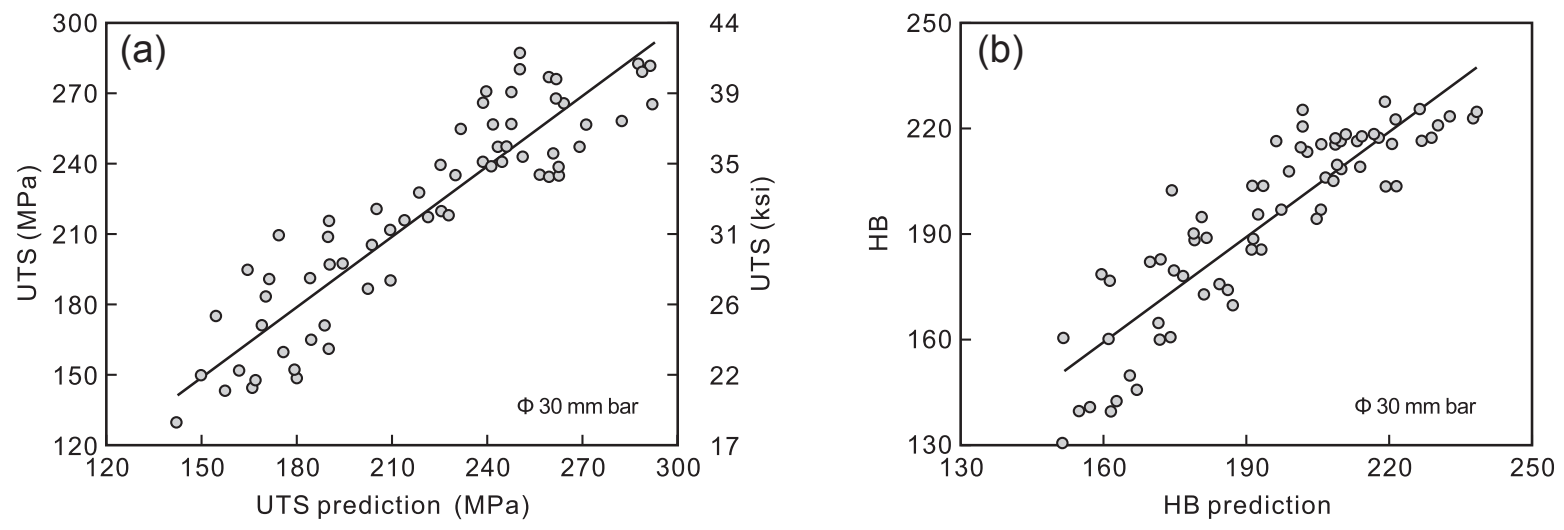

Fig. 36: Relationship between calculation and experiments on $30 \mathrm{~mm}$ diameter test bars ${ }^{[7]}$ : (a) tensile strength; (b) Brinell Hardness ${ }^{[7]}$

was actually the dendrite coherency point ${ }^{[10]}$. The beginning of primary and eutectic solidification, as well as the end of solidification, must be calculated from the 2 nd derivative ${ }^{[22]}$.

While prediction of many parameters is claimed, the data scattering is many times too large to be useful in the control of foundry processes. We believe this to be particularly true for prediction of shrinkage defects, where the outcome depends not only on metal quality, but also on gating and casting design. TA can only predict metal quality and shrinkage tendency in the test cup. Thus, the ideal solution should combine well-controlled TA measurements with computational models that can describe mold filling, calculation of graphite expansion, and gas evolution.

\section{References}

[1] Le Chatelier H. Z. Phys. Chem., 1887, 1: 396.

[2] Piwowarski E. High strength cast iron (Hochwertiges Gusseisen), Springer Verlag, Berlin, 1961.

[3] Piwowarski E. Giesserei, 1938, 25: 523.

[4] Loper C R, Heine R W, Reesman R W, et al. Thermal analysis of ductile iron. AFS Trans., 1967, 75: 541.

[5] Naro R, Wallace J F. Minor elements in gray iron. AFS Trans., 1970, 78: 229-238.

[6] De Sy A, Vidts J. Theoretical and applied metallurgical treatise (Traité de metallurgie structurale theorique et appliquée), Ed. Dunod, Paris, 1962.

[7] Suarez R, Larrañaga $P$, Udroiu A. Cooling curve (thermal) analysis. In: D. M. Stefanescu Ed., ASM Handbook, Vol. 1A: Cast Iron Science and Technology. ASM International, Materials Park Ohio, 2017: 149-159.

[8] Stefanescu D M. Thermal Analysis - Theory and applications in metal casting. Int. J. Metalcasting, 2015, 9(1): 7-22.

[9] Dawson S, Popelar P. Thermal analysis and process control for compacted graphite iron and ductile Iron. SinterCast, 2014, www.sintercast.com.

[10] Anjos V, Deike R, Silva Ribeiro C. The use of thermal analysis to predict the dendritic coherency point on nodular cast iron melts. Science and Technology of Materials, 2017, 29: 27-33.

[11] Stefanescu D M. Solidification of flake, compacted and spheroidal graphite cast irons as revealed by thermal analysis and directional solidification experiments. In: The Physical Metallurgy of Cast Iron, H. Fredriksson, M. Hillert eds. Elsevier, 1985: 151-162, doi:10.1557/PROC-34-151.
[12] Persson $P$ E, Ignaszak $Z$, Fransson $H$, et al. Increasing precision and yield in casting production by simulation of the solidification process based on realistic material data evaluated from thermal analysis (using the ATAS MetStar system). Archives of Foundry Eng., 2019, 19(1): 117-126.

[13] Charoenvilaisiri S, Stefanescu D M. The Possibility of prediction of graphite shape in Mg-treated irons by means of thermal analysis. In: Proceedings of the 7th Asian Foundry Congress, The Chinese Foundrymen's Association, Taipei, Taiwan, 2001: 91-100.

[14] Rabus D, Polten S. Gieserei Rundshau, 1972, 9: 1-8.

[15] Chen I G, Stefanescu D M. Computer-aided differential thermal analysis of spheroidal and compacted graphite cast irons. AFS Trans., 1984, 92: 947-964.

[16] Sparkman D A. Microstructure by thermal analysis. AFS Trans., 2011, 119: 413-420.

[17] Lekakh S N, Richards V L. Determining solidification parameters of alloy steels. AFS Trans., 2011, 119: 521-528.

[18] Ekpoom L, Heine R W. Thermal analysis by differential heat analysis (DHA) of cast iron. AFS Trans., 1981, 89: 27-38.

[19] Upadhya K G, Stefanescu D M, Lieu K, et al. Computer-aided cooling curve analysis, principles and applications in metal casting. AFS Trans., 1989, 97: 61-66.

[20] Kierkus W T, Sokolowski J H. Recent advances in CCA: A new method of determining baseline equation. AFS Trans., 1999, 107: 161-167.

[21] Dioszegi A, Svensson I. On the problems of thermal analysis of solidification. Mat. Sci. Eng. A, 2005, 413-414: 474.

[22] Alonso G, Larrañaga $P$, Sertucha J, et al. Gray cast iron with high austenite-to-eutectic ratio, Part I - Calculation and experimental evaluation of the fraction of primary austenite in cast iron. AFS Trans., 2012, 120: 329-335.

[23] Fras E, Kapturkiewicz W, Burbielko A, et al. A new concept in thermal analysis. AFS Trans., 1993, 101: 505.

[24] Barlow J O, Stefanescu D M. Computer-aided cooling curve analysis revisited. AFS Trans., 1997, 105: 349.

[25] Dioszegi A, Hattel J. Inverse thermal analysis method to study solidification in cast iron. Int. J. Cast Metals Research, 2004, 17: 311.

[26] Stefanescu D M, Lacaze J. Thermodynamics principles as applied to cast iron. In: D. M. Stefanescu Ed., ASM Handbook, Vol. 1A: Cast iron science and technology. ASM International, Materials Park Ohio, 2017: 31-45.

[27] Stefanescu D M, Katz S. Thermodynamic properties of ironbase alloys. ASM Handbook, Vol. 15-Casting, 2008: 41-55.

[28] Humphreys J G. Effect of composition on the liquidus and eutectic temperatures and on the eutectic point of cast irons. BCIRA J, 1961, 9 (5): 609-621. 
[29] Sillén R. Finding the true eutectic point - An essential task for efficient process control of ductile iron. Ductile Iron News, 2007, 2: $100-104$

[30] Donald W, Moore A. BCIRA J., 1973, Report No. 1128.

[31] Van der Perre W. Thermal analysis, principles and applications. Heraeus Electro-Nite, https://www.heraeus.com/en/hen/home_ heraeus_electro_nite/home_electro_nite.html.

[32] Suarez R, Sertucha J, Larrañaga P, et al. Active Mg estimation using thermal analysis: a rapid method to control nodularity in ductile cast iron production. Metall. Mater. Trans. B, 2016, 47B: 2744-2753.

[33] Kanno T, Fukuda Y, Morinaka M, et al. Effect of alloying elements on graphite and cementite eutectic temperature of cast iron. J. JFS, 1998, 70: 465-470.

[34] Kanno T, Iwami Y, Kang I. Prediction of graphite nodule count and shrinkage tendency in ductile cast iron with 1 cup thermal analysis. Int. J. Metalcasting, 2017, 11(1): 94-100.

[35] Kanno T, Kang I, Mizuki T, et al. Effect of S, Mn and Re on eutectic temperature of cast iron. J. JFS, 2001, 73: 441-446.

[36] Sparkman D A, Bhaskaran C A. Chill measurement by thermal analysis. AFS Trans., 1996: 969-976.

[37] Kanno T, Kang I, Fukuda Y, et al. Prediction of chilling tendency, graphite types and mechanical properties in cast iron, using three cups thermal analysis. AFS Trans., 2006, 114, paper No. 06-083.

[38] Ekpoom U, Heine R W. Thermal analysis by differential heat analysis (DHA) of cast iron. AFS Trans., 1981: 27-38.

[39] Bäckerud L, Chalmers B. Trans. Metall. Soc. AIME, 1969, 245: 309.

[40] Guo X, Stefanescu D M. Solid phase transformation in ductile iron-A benchmark for computational simulation of microstructure. AFS Trans., 1997, 105: 533-544.

[41] Sertucha J, Suarez R, Izaga J, et al. Prediction of solid-state structure based on eutectic and eutectoid transformation parameters in spheroidal graphite irons. Int. J. Metalcasting, 2006, 19(6): 315-322.

[42] Larrañaga $P$, Gutierez J M, Loizaga A, et al. A computer-aided system for melt quality and shrinkage propensity evaluation based on the solidification process of ductile iron. AFS Trans., 2008, 116: 547-561.

[43] Guesser W, Schroeder T, and Dawson S. Production experience with compacted graphite iron automotive components. AFS Trans., 2001: 01-071.
[44] Baer W. Chunky graphite in ferritic spheroidal graphite cast iron: formation, prevention, characterization, impact on properties: an overview. Int. J. Metalcasting, 2019, https://doi.org/10.1007/ s40962-019-00363-8.

[45] Stefanescu D M, Alonso G, Suarez R. Recent developments in understanding nucleation and crystallization of spheroidal graphite in iron-carbon-silicon alloys. Metals, 2020, 10: 221 , doi:10.3390/met10020221.

[46] Sertucha J, Suarez R, Asenjo I, et al. Thermal analysis of the formation of chunky graphite during solidification of heavysection spheroidal graphite iron parts. ISIJ Int., 2009, 49(2): 220-228.

[47] Chaudhari M D, Heine R W, Loper C R. Principles involved in the use of cooling curves in ductile iron process control. AFS Trans., 1974, 82: 379-386.

[48] Kweon E S, Roh D H, Kim S B, et al. Computational modeling of shrinkage porosity formation in spheroidal graphite iron: a proof of concept and experimental validation. Int. J. Metalcasting, 2020, doi.org/10.1007/s40962-020-00417-2.

[49] Svidró P, Diószegi A, Jöhnsson P G, et al. Determination of pressure in the extradendritic liquid area during solidification, J. Thermal Analysis and Calorimetry, 2018, 132: 1661-1667, https://doi.org/10.1007/s10973-018-7088-z(0123456789().,-volV)(0123456789,-().volV).

[50] Alonso G, Stefanescu D M, Suarez R, et al. Understanding graphite expansion during the eutectic solidification of cast iron through combined linear displacement and thermal analysis. In: Proc. 71st World Foundry Congress, Bilbao, Spain, 2014.

[51] Stefanescu D M, Qiu H Q, Chen C H. Effects of selected metal and mold variables on the dispersed shrinkage in spheroidal graphite cast iron. AFS Trans., 1995, 95: 189-197.

[52] Lekakh S N, Hrebec B. Solidification kinetics of graphite nodules in cast iron and shrinkage porosity. Int. J. Metalcasting, 2016, 10(4): 389-400.

[53] Persson P E, Udroiu A, Vomacka P, et al. ATAS $\AA$ as a tool for analyzing, stabilizing and optimizing the graphite precipitation in grey cast iron, In: Proceedings 69th World Foundry Congress, FICMES, Hangzhou, China, 2010.

[54] Erturk S O, Kumruoglu L C, Ozel A. Determination of feederless casting limits by thermal analysis in cast iron. Acta Physica Polonica A, 2017, 131(3): 370-373. 